\title{
La couronne ou la guirlande de la justification offerte au défunt ou au dieu pour le justifier ${ }^{1}$
}

\section{Dr. Salwa Kamel*}

\section{Introduction}

Les couronnes, les guirlandes, et les colliers végétaux que l'on plaçait sur le corps, le cou et la tête du défunt n'avaient au-début qu'un but ornemental ; on couronnait le mort, d'après les textes, comme pour les fêtes. Mais bientôt cette ornementation florale reçut un caractère religieux ou symbolique.

Le nom de la couronne et la guirlande de la justification était ( $\mathrm{mAH}$ $n$ mAaxrw) $)^{2}$ dans toutes les époques.

La guirlande a cité probablement pour la première fois dans les textes des Pyramides sous le nom wAH n.k tp. K $^{3}$.

Dès la $22^{\mathrm{e}}$ dyn. surtout à l'époque grecque, on ne trouve presque aucune momie sans cette couronne, qu'elle soit faite de fleurs naturelles ou certainement indiquée en couleurs ${ }^{4}$.

La couronne symbolise le nouvel caractère qui a remarqué victorieusement l'expression du jugement ${ }^{5}$.

\section{*مدرس بكليه الاثار جامعة القاهرة}

${ }^{1}$ La couronne de la justification a attiré l'attention de "W.Pleyte" en 1884 qui a fait un article sur les exemplaires de couronnes et de guirlandes végétales trouvées sur les momies et conservées dans les musées et le chapitre 19 du Livre des Morts.

W.Pleyte, «La couronne de la justification », dans : Actes du sixième Congrès International des Orientalistes, Leiden (1885), 1-30, pl. I-XXV.

« Ph.Derchain » a publié un article sur la couronne de la justification. Essai d'analyse d'un rite ptolémaïque dans : le Chronique d'Égypte 30, n 60 (1955), 225-287.

D.Jankuhn, “Kranz der Rechtfertigung”, dans: LÄ III, (1980), 764.

${ }^{2}$ Wb II, 31 (5); P.Wilson, OLA 78, (1997), I, 397.

${ }^{3}$ LÄ III, 764. Pyr.1213 d.

${ }^{4}$ L.Keimer, «L'arbre Tr.t $=-\ell$ est il réellement le saule égyptien (Salix Safsaf Forsk) ? », dans : BIFAO 31, (1931), 206.

R.Germer, "Weide", dans: LÄ VI, (1986), 1164.

${ }^{5}$ Ph.Derchain, CdE 30, 231 sq. = 
Un texte magique (Leide, 347, 8, 10-11), appartenant au Nouvel Empire qui cite la couronne de la justification concernant de la victoire contre les ennemis ${ }^{6}$ :

smAzxrw.k r xftjw.k ....... mAH n mAzxrw r.k "Tu seras justifié contre tes ennemis...avec la couronne de la justification sur toi".

Le caractère de $m A z x r w$ s'applique à $R^{c}$ lui-même lorsqu'il triomphe de son ennemi Apophis ${ }^{7}$.

Aussi qu'Osiris triomphant et décoré de guirlandes de fleurs avait laissé la salle de jugement d'Héliopolis, on souhaitait que le mort parût devant Osiris, juge des morts, paré de la «couronne de la justification $» m A H n$ mAarrw ${ }^{8}$.

Cette ancienne tradition de couronner les morts c'est continuée jusqu'à l'époque des Grecs et des Romains, la couronne se donne à celui qui brille dans les sciences, ou dans les arts, ou à la guerre?

\section{Les différentes écritures de la couronne mAH :-}

\begin{tabular}{|c|c|}
\hline$\infty$ & E I, 62-63. \\
\hline$\Rightarrow$ & E V, 103-104. Philae, 274. \\
\hline acs & $\begin{array}{l}\text { E V, 67-68. E VIII, 56-57. E VIII, 119. D V, 83-84. E III, 141- } \\
\text { 142. E VI, 287-288. E VII, 308-309. D X, } 16 .\end{array}$ \\
\hline 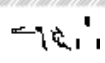 & E I, 236. E II, 71. E I, 107-108. \\
\hline $\mathscr{O}$ & E IV, 355-356. Opet, 291-292. \\
\hline$a x$ & E VIII, 79-80. \\
\hline 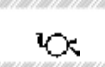 & D X, 240. D II, 157. Dendour, 8, 45 \\
\hline 8 & E VII, 275-276. Philae, 305. \\
\hline
\end{tabular}

= D.M.Mostafa, «L'usage cultuel du bouquet et sa signification symbolique », dans :

Hommages à Jean Leclant, BE 106/4, (1994), 244.

${ }^{6}$ W.Pleyte \& F.Rossi, Papyrus de Turin, 1869-1876, pl. 123, 1.

Sur la «justification“" et la victoire sur les ennemis :

J.Yoyotte, «Enquètes Potasimto de Pharbaïthos et le titre grand combattant- maître du triomphe », dans : CdE 28, (1953), 101-106.

${ }^{7}$ CT V, 67, 393. CdE 30, 239. LÄ III, (1980), 764.

${ }^{8}$ L.Keimer, BIFAO 31, 205-206.

${ }^{9}$ W.Pleyte, Ibid., 11, 17-18. 
1 - ماسات في آثار الوطن العربي

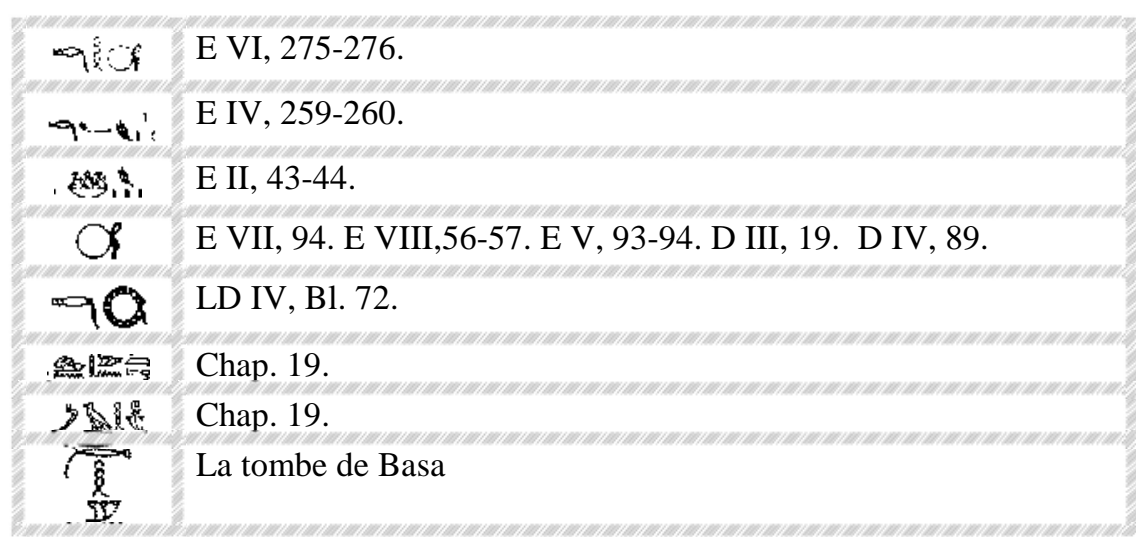

La justification était souvent figurée par des fleurs nouées en couronnes et en guirlandes qui jouaient un rôle analogue à celui des bouquets ${ }^{10}$.

Ce symbolisme passa dans le signe ${ }^{*}$, est identique à $\varpi_{0}$; il remplace ce dernier surtout en origine à la couronne de la justification $\mathrm{mAH} n \mathrm{mAz} \times \mathrm{xw}^{11}$.

\section{La fabrication de la couronne}

On coupe dans une feuille de palmier doum, un petit ruban, dont la largeur $3 \mathrm{~mm}$. et la longueur assez très longue.

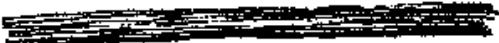

Pleyte, La couronne, pl. XXIV.

On tient une feuille d'olivier ou de perséa, que l'on plie en deux, presque par la moitié.

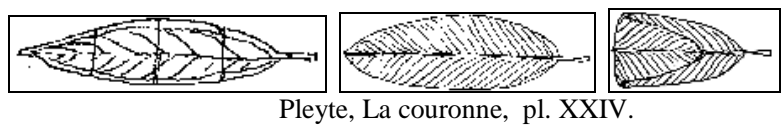

On tient ensuite un pétale d'une fleur, et on la pose dessue, enfin on y colle la bande, et les feuilles sont pliées plusieurs fois sur elles mêmes, puis posées l'une sur l'autre, sur un ruban de feuilles de

\footnotetext{
${ }^{10} \mathrm{Ph}$. Derchain, «La Visite de Vespasien au Sérapeum d'Alexandrie », CdE 56, (1953), 271. L.Keimer, «Egyptian formal bouquets », dans : SLL 41, (1925), 145.

${ }^{11}$ W. Spiegelberg, «Varia », dans : Rec de trav. 26, (1904), p. 49.

Wilson, OLA 78, I, 397.
} 
I - مراسات في اثثار الوطن العربي

palmier, fin comme une corde, attachée enfin dans un cercle avec les ornements.

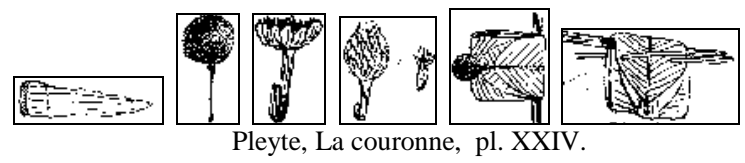

On prend deux cordes très minces, découpés dans une feuille de palmier doum, et l'on s'en sert pour coudre les feuilles ensemble.

Quand le ruban est très long, on relie les boues en façon d'un chaton ou d'un nœud, on noue rarement une corde ou cordelette composée de plusieurs cordes fines.

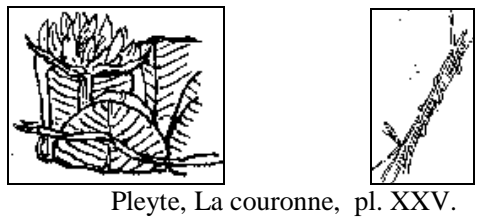

On distingue l'habitude de faire des couronnes calquées sur le carton, puis dorés et les feuilles sont crayonnées en noir ${ }^{12}$.

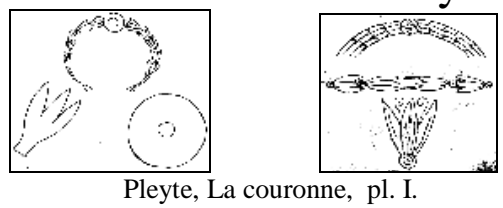

Les matières qui entrent dans la fabrication de la couronne :-

Les fleurs et les feuilles nouées en couronnes que l'on a découvertes à Deir el-Bahari sont en feuilles de saule ou de perséa pliées; on avait inspiré dans les plis des feuilles le calice ou les pétales de fleurs de différents groupes ${ }^{13}$.

Le lotus $^{14}$ est très attaché au défunt à la conception de justification, ainsi le signe de cette fleur probablement utilisé pour déterminer ou même écrire $m A z \times r w^{15}$, le mythe osirien montre encoure lier cette couronne au texte de la conformité royale.

\footnotetext{
${ }^{12}$ Ibid., 4-6.

${ }^{13}$ Ibid., 7.

${ }^{14}$ M.L. Ryhiner, L'Offrande du lotus dans les temples égyptiens de l'époque tardive, Rites égyptiens VI, Bruxelles, (1986), 219 sq.

${ }^{15}$ W.Erichsen, “ $\cong$ l 278.=
} 
Les feuilles n'étaient pas toutes du même groupe ; on y fait une allusion sur des feuilles d'olivier et d'un groupe de gloire ${ }^{16}$, les feuilles de vigne, les grains du blé poussés, et les fleurs de Chrysanthèmes sauvages.

Les exemplaires de couronnes végétales trouvées sur les momies :-

Les momies d'Amenemhet et sa femme étaient ornées de guirlandes. ${ }^{17}$

La momie d'Amosis, $18^{\mathrm{e}}$ dyn. entourée de guirlandes de fleurs selon l'usage préservant des Égyptiens de l'époque thébaine, aussi la momie d'Aménophis I, entourée de fleurs ${ }^{18}$, en plus d' Aménophis II ${ }^{19}$.

Toutes ces fleurs, ces feuilles, ces couronnes découvertes en même temps que les momies ne paraissent donc pas dater de l'ensevelissement, mais probablement ont été posées sur les momies à l'époque de la $22^{\mathrm{e}}$ dyn., lorsqu'on les a transportées dans la cachette de Deir el-Bahari ${ }^{20}$.

Le cercueil contenant la momie de Toutankhamon était orné de guirlandes et de colliers, mais d'autre part le masque en or porte sur la tête ou, plus exactement, autour de l'uræus et de la tête de vautour qui surmontent le front, une véritable petite couronne de la justification $^{21}$. Fig. 1

=B.Geßler-Löhr,"Zur Schreibung von mAzxrw mit der Blume”, dans : GM 116, (1990), 25-43.

Cette habitude graphique, peut-être d'origine héliopolitaine, apparaît à la fin du Moyen

Empire et semble être réservée aux défuntes après la période amarienne.

R.Hannig, Ägypt. Wörterbuch II, I, 1001-1002.

P.P.Koemoth, « Osiris et les arbres, Contribution à l'étude des arbres sacrés de l'Égypte ancienne, dans : Egyptiaca Leodiensia 3, (1994), 34.

${ }^{16}$ R. Germer, Flora des pharaonischen Ägypten, dans: Sonderschrift 14, (1985),150-151.

Sur l'olivier, arbre royal et millénaire en Grèce

P.Ellinger, dans : Dictionnaire des mythologies, Paris 1981, I, 72b.

${ }^{17}$ Davies-Gardiner, The Tomb of Amenemhet No. 82, 1915, 111.

${ }^{18}$ CdE 30, 225.

${ }^{19}$ H.Carter, "Report on the Robbery of the Tomb of Amenothes II, Biban el Moluk", dans: ASAE 3, (1903), 121.

${ }^{20}$ Pleyte, La couronne, 7.

${ }^{21}$ H.Carter, The Tomb of Tut-Ankh-Amen, t. II, 1927, pl. LXVII.= 
La décoration de fleurs et de feuilles naturelles de Sen Nefer se composait de cinq étages de gerbes et de colliers, parmi elles une guirlande de feuilles de vigne ${ }^{22}$. Fig. 2

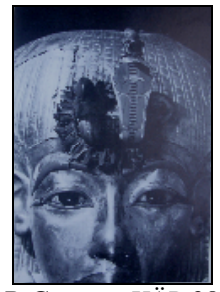

Fig. 1. R.Germer, $H \ddot{A B}$ 28, Taf. I.

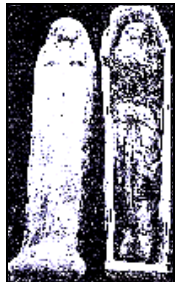

Fig. 2.B.Bruyère, FIFAO 6, 2, pl. 4, 6

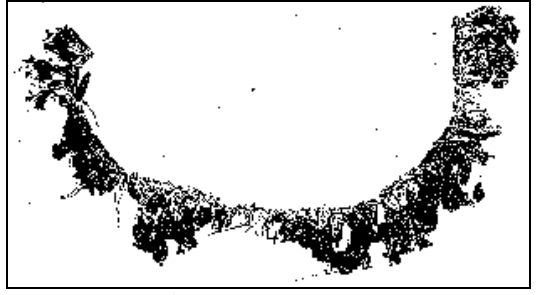

Le Musée de Berlin possède une guirlande composait de feuilles naturelles ${ }^{23}$. Fig. 3

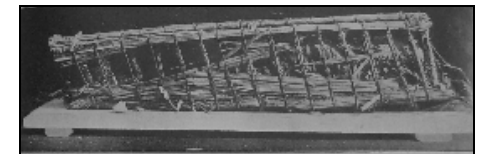

Fig. 3. O.Rubensohn-Knatz, dans : Z̈̈S 41, 11, Abb.2. Berlin, Inv. 19538-44.

Le Musée d'agriculture au Caire possède des exemplaires en des différentes feuilles. Fig. 4-8

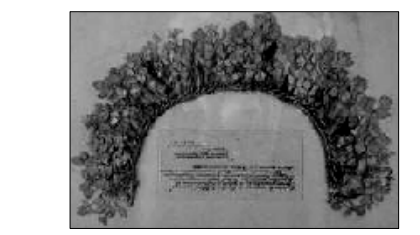

Fig. 4. Les grains du blé poussés, Thèbes,

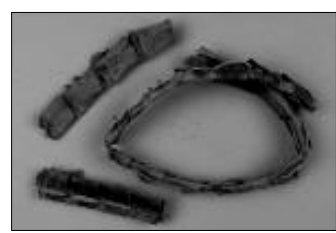

Fig. 5. Les restes de guirlande de feuilles de perséa, Nouvel Empire.

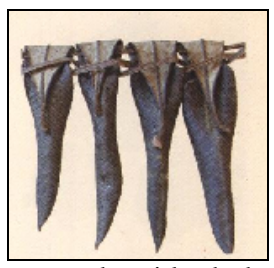

Fig. 7. Les restes de guirlande des feuilles d'olivier, Thèbes, Nouvel Empire

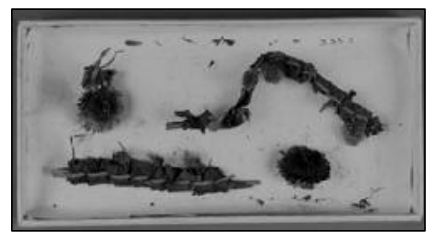

Fig. 6. Les restes de guirlande de fleurs des Chrysanthèmes sauvages et feuilles de perséa. Thèbes, Nouvel Empire.

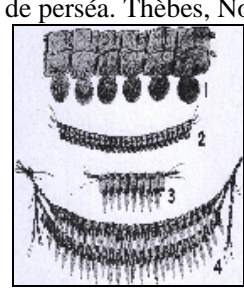

Fig. 8.Guirlandes au saule Thèbes, Nouvel Empire

=L.Keimer, dans : BIFAO 31, (1931), 206.

R.Germer, "Die Planzenmaterialien aus dem Grab des Tutanchamun",dans:Ḧ̈B 28,(1989),5-6.

${ }^{22}$ B.Bruyère, Deir el Medineh, FIFAO 6, 2, Le Caire 1929, 52, pl. 4, 6.

${ }^{23}$ O.Rubensohn-Knatz, " Bericht über die Ausgrabung bei Abusir el Mäläq im Jarhe 1903 ,, , dans : Z̈̈S 41, (1904), 11, Abb.2. 
Dans un cas, unique, le feuillage d'une couronne de momie est doré ${ }^{24}$. Fig. 9.

Les couronnes végétales qu'on trouve sur les momies et qui sont représentées sur certains monuments privés expriment un espoir de vie $^{25}$. Fig.10.

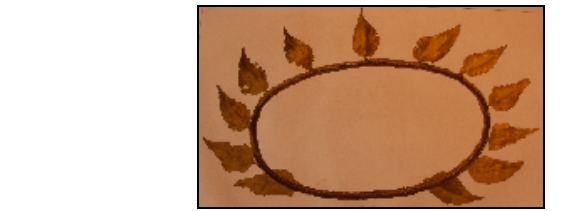

Fig. 9. S.Birch, Facsimilie, fig. 2.

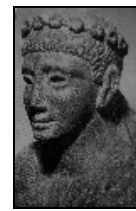

Fig. 10. A.Rowe, dans : $A S A E$ 40, fig. 2.

\section{Les textes funéraires qui citent la couronne de la justification :-}

Les textes que se trouvent dans le Livre de Morts semblables aux Rituel de Rougé, le papyrus funéraire de Leide 16, et le papyrus $74 ; 65-43$.

Dans le Chapitre 19 du Livre des Morts, le défunt, reconnu pur et vainqueur de ses ennemis devant tous les tribunaux, reçoit la marque de son triomphe: la couronne la de justification ${ }^{26}$.

\section{Formule de la couronne de justification ${ }^{27}$ :}

rAn mAH n mAaxrw

Chapitre de la couronne de justification.

Paroles dites par Osiris N. : «Ton père Atoum a ceint ton front de cette belle couronne de justification, comme vit l'aimé des dieux, vis éternellement! Osiris, le chef de l'occident, te proclame victorieux de tes ennemis et ton père Geb t'a donné son héritage. Viens triomphant et justifié. Horus fils d'Isis et d'Osiris est sur le trône de son père et Ré abat tes ennemis. Il t'a attribué le double pays entier. Atoum a décrété, et l'ennéade a répété la belle condition de victorieux d'Horus fils d'Isis, fils d'Osiris, éternellement et de l'Osiris $N$. éternellement et à jamais».

\footnotetext{
${ }^{24}$ S.Birch, Facsimilie of two papyri found at Thebes, 1863, pl. face à la p. 26, fig. 2 .

${ }^{25}$ A.Rowe, « Newly-identified monuments in the Egyptian Museum showing the deification of the dead together with brief details of similar objects elsewhere », dans: ASAE 40, 18, fig. 2. CdE 30, 230.

${ }^{26}$ S.Sauneron, "Le Chancelier du Dieu" [...] dans son double rôle d'embaumeur et de prêtre d'Abydos », dans : BIFAO 51, (1952), 163. \& Urk. V, 137-138.

${ }^{27}$ J.L.de Cenival, Le livre pour sortir le jour, le Livre des Morts des anciens égyptiens,1992,57.

P.Barguet, Le Livre des Morts des anciens égyptiens, 1967, 67-68.
} 
Cette formule sur la couronne divine placée devant le mort, en même temps qu'on met des pastilles d'encens sur la flamme (de l'encensoir) pour l'Osiris N.; (c'est) faire qu'il soit proclamé victorieux contre ses ennemis morts ou vivants; et il sera parmi les suivants d'Osiris, et on lui donnera des cruches (de bière) et des pains devant ce dieu.

(Quand c'est) dit sur toi à la pointe de l'aube, c'est une grande protection véritablement efficace, indéfiniment.

\section{Chapitre 20}

(Autre chapitre de la couronne de la justification).

"O Thot qui as proclamé victorieux Osiris de ses ennemis de l'Osiris $N$. devant le tribunal de chaque dieu et de chaque déesse, devant le grand tribunal qui est à Héliopolis ».

Thot, proclame victorieux l'Osiris N. contre ses ennemis, devant le tribunal de chaque dieu et de chaque déesse.

On voit les chapitres 19 et 20, la vignette, qui représente une personne devant Atoum recevant la couronne de la justification, se rencontre pour la première fois dans un texte du temps des Psamétiques.

Le texte est probablement de beaucoup postérieur ; en tous cas je ne l'ai rencontré que dans les livres de l'époque des Grecs et des Ptolémées $^{28}$.

De Rougé a traduit le titre comme suit : «Chapitre de la couronne de justification ».

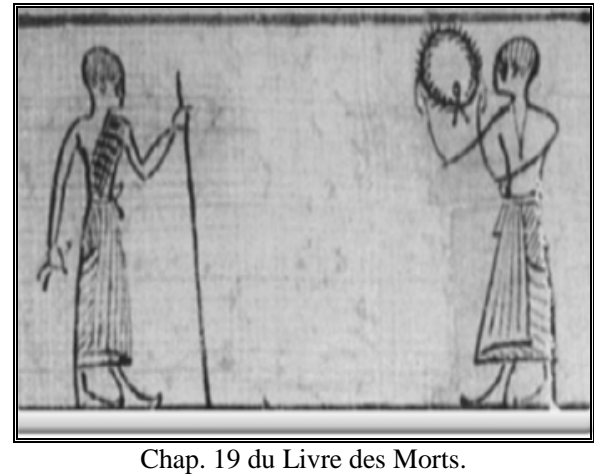

Chap. 19 du Livre des Morts.

\footnotetext{
${ }^{28}$ W.Pleyte, op.cit., 7.
} 
- مراسات في اثار الوطن العربي •
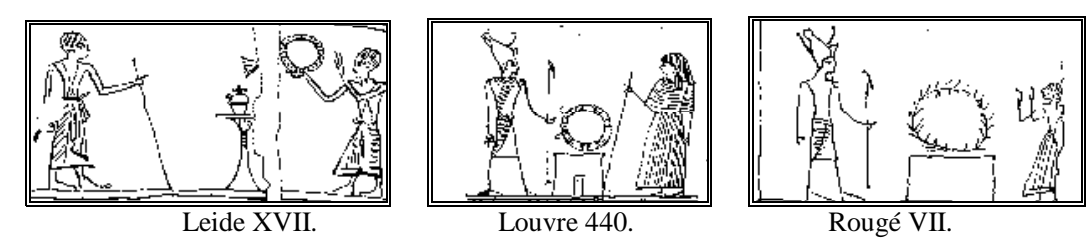

\begin{tabular}{|c|c|}
\hline $\begin{array}{c}\text { Chap. } 19 \\
\text { Rougé VII. }\end{array}$ & 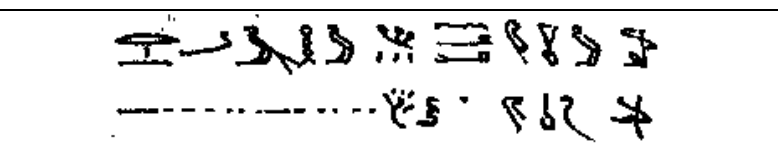 \\
\hline \multicolumn{2}{|l|}{ Leide XVII. } \\
\hline Chap. 19 & \multirow{3}{*}{ 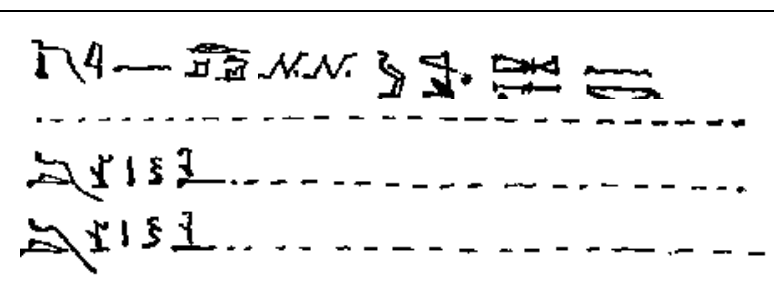 } \\
\hline Louvre 440. & \\
\hline Rougé VII. & \\
\hline Chap. 19 & \multirow{4}{*}{ 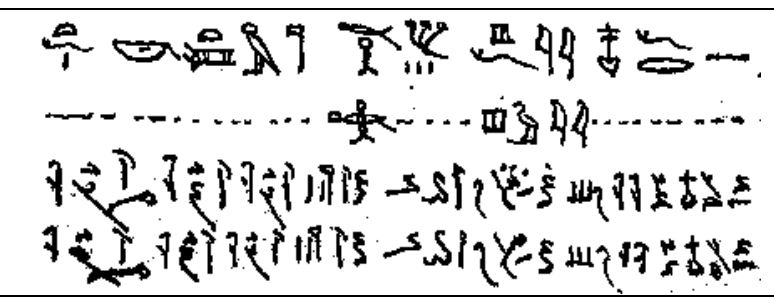 } \\
\hline Louvre 440. & \\
\hline Rougé VII. & \\
\hline Leide XVII. & \\
\hline
\end{tabular}

Où et quand se présente la couronne de la justification à l'audelà au défunt ?

La couronne de la justice se donnait au défunt, à l'entrée de l'occident. Il y avait là un sycomore, dans lequel demeurait la déesse du ciel, Nout, qui donnait à boire de ces eaux célestes, il recevait la couronne sous ce sycomore dans le chapitre 59.

La couronne est donc pour le défunt le signe qu'il a été proclamé juste après la confession, ainsi qu'on le lit au chapitre $97^{29}$.

${ }^{29}$ W.Pleyte, La couronne, 9-10. 
1 · راسات في اثثار الوطن العربي

Le rite ptolémaïque d'offrande de la couronne de la justification se présente sous une forme complexe, où entrent des éléments qui appartient à Horus et à Osiris.

Il a deux raisons de fixer le rite essentiel à Horus:-

1- L'offrande à Horus est la plus fréquente.

2- Dans les textes des Pyramides ${ }^{30}$, la justification concerne d'abord et primitivement Horus et ce n'est que secondairement qu'on la donne à Osiris.

Il ne s'agit pas d'un rite d'origine funéraire comme l'existence du chapitre 19 du Livre des Morts et les couronnes de momies ${ }^{31}$.

\section{La couronne de la justification offerte à Horus :-}

Doc. $\mathbf{n}^{0} 1$

Titre: Ts mAH (n mAaxrw) Dd mdw pr.k m wpt.k mdw.k m mAaxrw mdw qn xtjw.k nAnbw I wnw Hrj wpi.k sni Hrj dmD dniwtj.k

«Nouer la couronne (de la justification). Discours : tu sors de ton procès, ta voix est juste tandis que la voix de tes ennemis est condamnée. Les seigneurs d'Héliopolis jugent ton procès et il leur plaît de réunir les deux parts qui te reviennent ».

Le Roi : nswt-bj.tt.P, nb tAwy. N, @r kAnxt (r)st it.f iTpp nbw m mAzxrw Dd mdw in mn n.k mAH pwj $n$ mAaxrw Ts.n.i r-HAt anx.k iT.n.k wDa ryt.k Hr nst Gbb xr it.f Wsir Wnnfr mAaxrw

"Le roi de Haute et de Basse Égypte. P, Seigneur des deux terres. N, Horus, taureau puissant (à) la place de son père, qui reçoit tous les pays en triomphateur. Discours : Accepte, je te prie cette couronne de la justification, je la noue autour de la tête pour que tu vives, quand tu reçois ta couronne de Haute Égypte sur le trône de Geb, en présence de ton père Osiris Onnophris le justifié» ${ }^{32}$.

\footnotetext{
${ }^{30}$ R.Anthes, "The original meaning of mAaxrw", dans: JNES 13,(1954), 51.

${ }^{31}$ CdE 30, 234.

${ }^{32}$ Edfou I, 62-63, pl. XVI.
} 
1 - مراسات في اثثار الوطن العربي

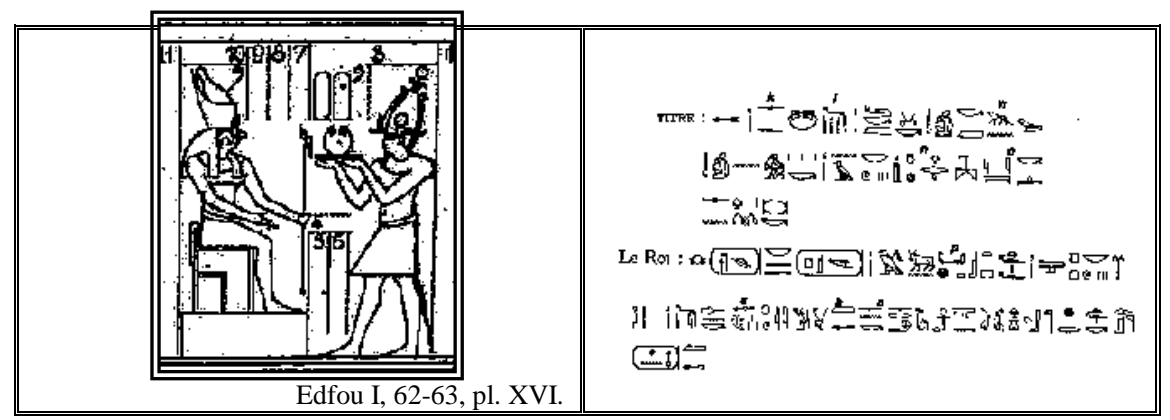

Doc. $\mathbf{n}^{\mathbf{0}} 2$.

Titre : Ts mAH n mAzxrw n it.f ir.f di anx

«Nouer la couronne de la justification pour son père, pour qu'il soit doué de vie ».

Le Roi : di.f Awt nb snb nb di.f anx wAs nb mi RaDd mdw mn n.k mAH nfr n mAz xrw Ts.n.k r-HAt xaHr st nt it.k iT. n.k tAwy mAz [xrw]

"Qu'il donne toute joie, toute santé !qu'il donne toute vie et prospérité, comme $R^{c}$. Discours : Accepte, je te prie, la belle couronne de la justification que tu as nouée autour de la tête lorsque tu apparais sur le trône de ton père, quand tu as reçu les deux Égypte en [triomphateur] » ${ }^{33}$.

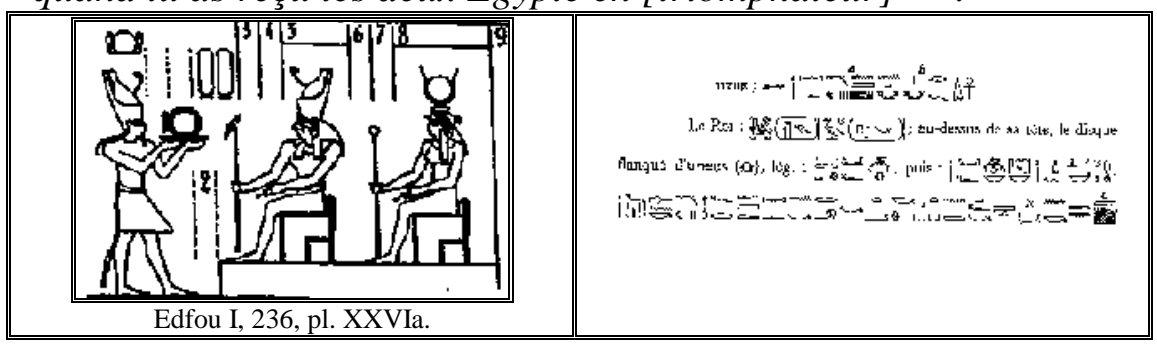

Doc. $n^{0} 3$.

Titre : Ts mAH n mAaxrw. Dd mdw nxt BHdtj m P Msn : iT. n.i tAwy n mAaxrw iT. n.i mAH tAwy m mAaxrw $n$ it.f W sir xawt Hr nst G bb pt Hr tp.f tAXr rdwy.f pDwtpsDt dmD Xr Tbtyw.f

« Nouer la couronne de la justification. Discours : Behedety puissant à Pe et à Mesen : il reçoit les deux Égypte en triomphateur. Il reçoit la couronne d'Égypte de son père Osiris, ou moment où il apparait sur le trône de Geb. Le ciel est au-dessus de lui, la terre est sous ses pieds, les Neuf Arcs sont réunis sous ses sandales».

${ }^{33}$ Edfou, I, 236, pl. XXVIa. 
Le Roi : mnx nTr @r dm Hnty Hr st it.f rdit Awt n aw.t.f @r anx Hwnw nb Hknw.f rdi PsDt m Awt aA mAaxrw pr m wpt.f nb Hr DADAt imyw I wnw Nbty pHty Hnt wpt wDa mdw

«Dieu Excellent. Horus aux cornes acérées sur le trône de son père, qui donne la joie à son troupeau, Horus vivant. Adolescent, Seigneur des louanges que son Ennéade donne en offrande, Grand Justifié sorti de tous ses procès devant le Grand Conseil qui est à Héliopolis, Nebty, redoutable qui châtie l'insolent, qui le juge après délibération ${ }^{34}$.

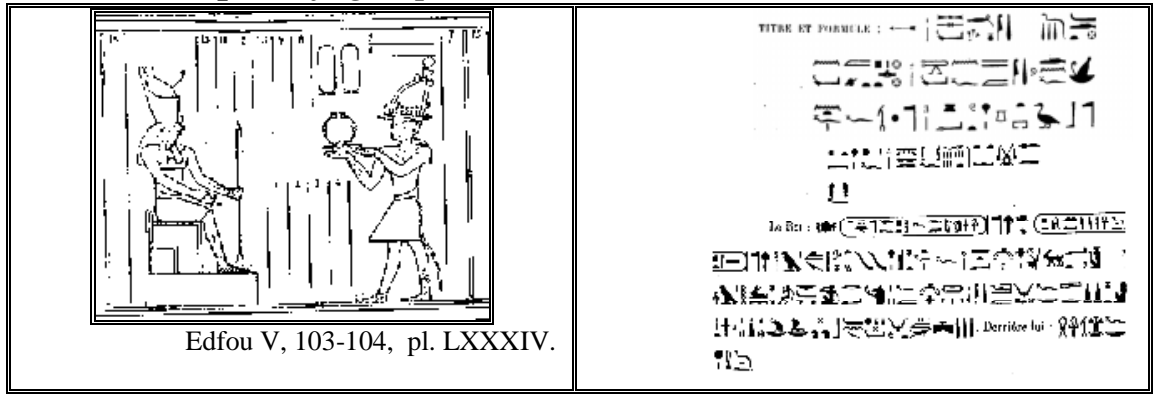

Doc. $n^{0} 4$.

Titre : di mAH n mAaxrw Dd mdw in mAH $r$ tp.k m Drt Tm sSdm dr.k m ibr mD HkAn.k tAwy iT.n.k wrrt wbn Hr nst it.k pt Hr tp.k tA Tbtyw.k nTrw ib sni

«Offrir la couronne de la justification. Discours : la couronne pour ta tête est de la main d'Atoum, tandis que tu es vêtu de la bandelette et que tu es oint d'Iadanum. Tu gouvernes les deux Égypte, après avoir reçu la couronne de Haute Égypte lorsque tu apparais sur le trône de ton père, tandis que le ciel est au-dessus de toi, que la terre est sous tes sandales et que les dieux (en) ont le cour heureux».

Le Roi : nTr mrj mwt.f snn n @r rdit n Qmt int nw mAzxrw Dd mdw iw n.k Hr.k BHdty nb M sn iTwrrt Hr st it.f Dd mdw In n.k mAH n mAaxrw r tp.k Ts n.k it.k it nTrw twt nTr m wpt.f

«Le dieu chéri de sa mère, l'image d'Horus, qui donne ... à l'Égypte lorsqu'il la parcourt en triomphateur. Discours: Je viens à toi. Behedty seigneur de Mesen, qui reçoit la couronne de Haute Égypte sur le trône de son père. Je t'apporte la couronne de la justification pour ta tête (cette couronne) qu'a tressée pour toi ton père, le père des dieux, en ta qualité de dieu [sorti] de son procès» 35 .

\footnotetext{
${ }^{34}$ Edfou, IV, 103-104, pl. LXXXIV.

${ }^{35}$ Edfou, IV, 355-356, pl. CVI.
} 


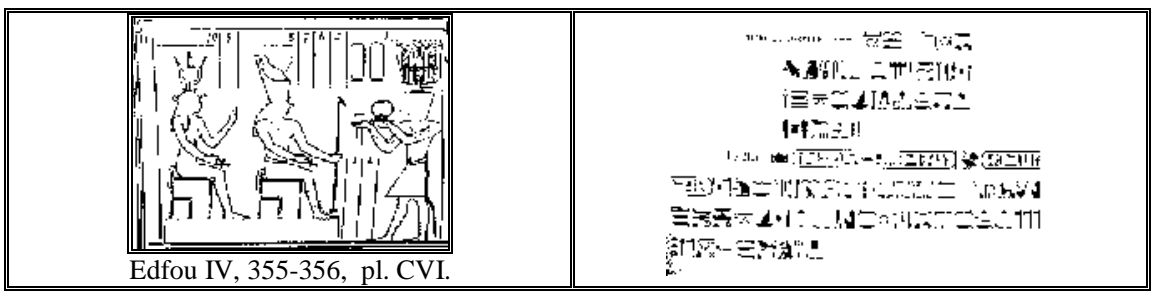

Doc. $n^{0} 5$.

Titre : ms mAH n mAaxrw Dd mdw : kA[......]

"Présenter la couronne de la justification. Discours : Gloire [......] ».

Le Roi : Wnn///Hr nst.f m HnwnDm mryt Hr ///////mAH Hr.s kA-aA@r Hr / / / / / it.f ir.n.f tAm / / / /

«Or[le roi de Haute et de Basse Égypte] est sur le trône un jeune homme dont l'amour est doux, en train de [Présenter] la couronne, de réjouir le dieu, d'élever [la couronne vers celui à qui elle appartient, car il est Haren] dotés, il met la terre sous sa protection» ${ }^{36}$.

\begin{tabular}{|c|c|}
\hline Edfou $\mathrm{V}, 67-68, \mathrm{pl.} \mathrm{CXIV}$. & \\
\hline
\end{tabular}

Doc. $\mathbf{n}^{0} 6$.

Titre : [ms mAH n] mAzxrw Dd mdw... sAWsir ... ( )niwt.f wADt nxt HAt niwt(.f) iT HDt nTrw ib.sn nDm mAaxrw.k xr DADAt

« [Présenter la couronne de] la justification. Discours ... fils d'Osiris... (qui préside) à sa ville, tandis qu'il est florissant et vainqueur à la tête de (sa) ville, prenant la Haute Égypte. Les dieux et les déesses ont le cour satisfait. Tu es justifié en présence du Conseil ».

Le Roi : Ink Sm r.k nb niwt iTHDt wr Sfyt n @t Ws... In n.k mAH nfr mAazrw, sqAy.s r.k twt kAwr.k (@t K Ak spd Hnty dm Hnty

«Je suis venu vers toi. Seigneur de la ville, qui prends la Haute Égypte, dont la dignité est grande dans @t Ws...Je t'apporte la belle couronne de la justification, je l'élève devant toi. Tu es le grand taureau de (@t KAk, aux cornes pointues, aux cornes acérées ${ }^{37}$.

\footnotetext{
${ }^{36}$ Edfou, V, 67-68, pl. CXIV.

${ }^{37}$ Edfou, V, 190-191, pl. CXIX.
} 


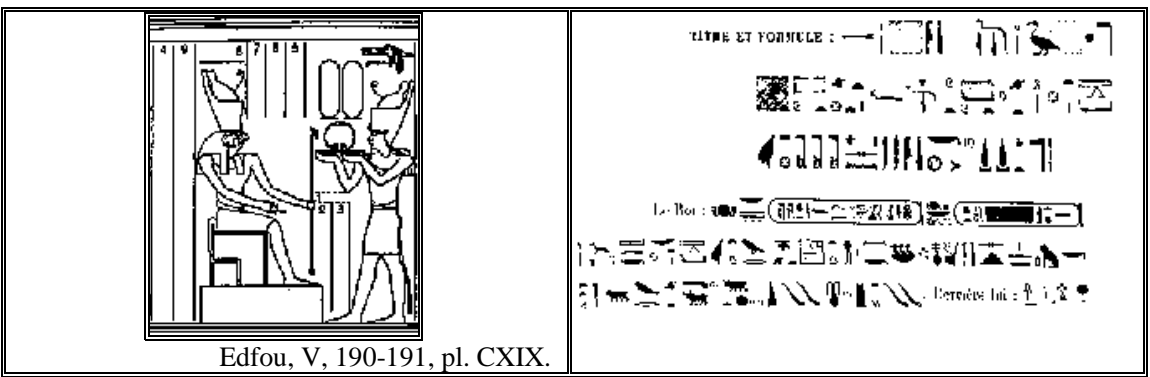

Doc. $n^{0} 7$.

Titre : ms mAH n mAzxrw Dd mdw in : kAr.k @r nxt m WTst @r xa Hm.k Hr st it.k

«Apporter la couronne de la justification. Discours: Gloire à toi Horus vainqueur à Edfou, quand ta majesté apparaît sur le trône de ton père ».

Le Roi : Wnn nsw Hrt nst.f m(i) bH wnw nfr nb aAHr kAt mAH Hr imntt iAbtt.f Hr sqA mDH n nb.f sw mi @r...n.f pDwt-psDt in kiyw.n.f xftyw.f ( ) Xr.f

«Or le roi était sur son trône comme un beau jeune homme très content, en train de présenter la couronne à sa gauche et à sa droite, d'élever la couronne vers son maître. Il est comme Horus lorsqu'il... Les Neuf Arcs, lorsqu'il blessé ses ennemis (tombés) sous lui ${ }^{38}$.

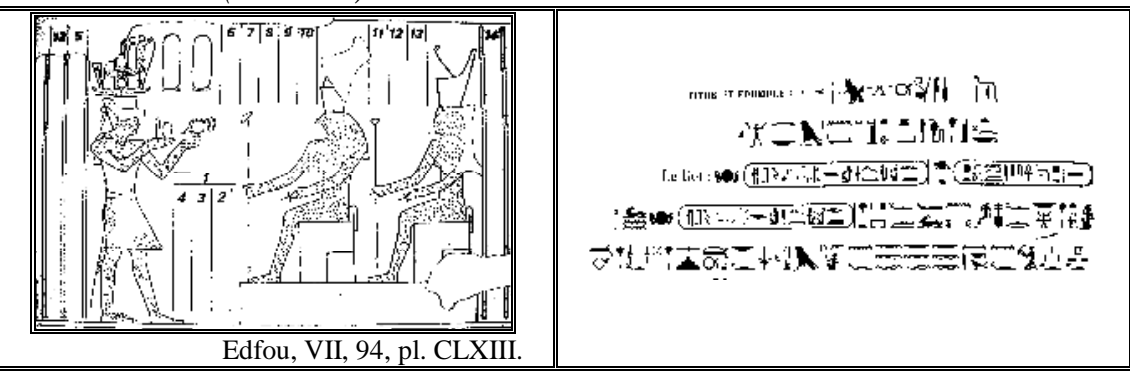

Doc. $\mathbf{n}^{0} 8$.

Titre : ms mAH n mAaxrw Dd mdw: mAH n mAzxrw m-HAt tp.k n anxw wsr Hm.k xnt anxw mAaxrw.k HAt @rw NTrw NTryt m DADAt sDm mdw wHm .k n nw iw fdw Hknw xr xftyw.k sxr

«Apporter la couronne de la justification. Discours: la couronne de la justification est sur ta tête, pour que vive et soit puissante ta majesté à la tête des vivants. Tu es justifié devant les dieux et les déesses, devant le conseil qui écoute les plaidoyers. Tu répètes par quatre fois les louanges, tes ennemis tombent, ils sont renversés ».

Le Roi : nTr pn... mri mwt.f ...HAt nst $n$ BHdt Hr ir mAđt Hr bHn grg dr isft m tAw sw m nb spd Hntj Ts.n.f mAzxrw m nxt tAwy

${ }^{38}$ Edfou, VII, 94, pl. CLXIII. 
« Ce dieu ... chéri de sa mère... qui préside au trône d'Edfou, en train de faire la justice, de réprimer le mensonge, de repousser les péchés dans les pays. Il est le seigneur des cornes pointues; il reçoit la justification dans les deux Égypte» ${ }^{39}$.

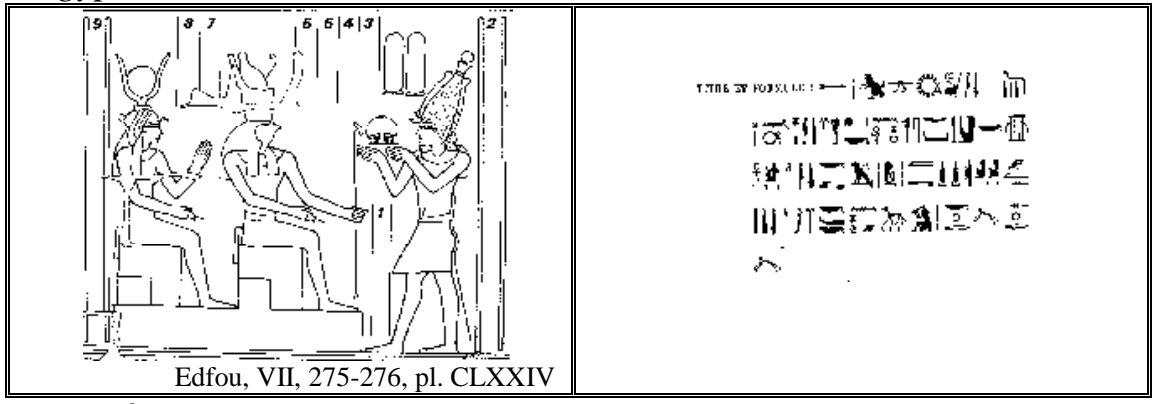

Doc. $\mathbf{n}^{0} 9$.

Titre : di mAH n mAzxrw Dd mdw: mAH r tp.k wTs.f HAt.k Ts.n.k tAwy m mAaxrw fdw n niwty pt $\mathrm{n}$ hm.n

kAk Dr xa.k Hr st it.f pt Hrj tp.k tAXr rdwy.k pDwt-psDt dmD Xr Tbtyw.f

« Présenter la couronne de la justification. Discours: la couronne est sur ta tête, elle pare ta tête lorsque tu reçois les deux Égypte en triomphateur. Les quatre coins du ciel acclament ton ka dès que tu apparais sur le trône de ton père. Le ciel est au-dessus de toi, la terre est sous tes pieds, les Neuf Arcs sont réunis sous tes sandales ».

Le Roi : @r anx N.nb BHdt sHtp sxmw m sxm.sn Nbty wr pHty bHn grg dr awAy dr xsf.n Hr n nxt @r nwb aApHty Ts tAwy m nxt qn HDt dSrt

«Horus vivant N., Seigneur d'Edfou, qui apaise les puissants dans leurs temples, "Maîtresse de la double couronne», redoutable, qui anéantit le menteur, qui supprime le voleur, qui repousse le révolté. Horus d'or redoutable qui prend les deux Égypte en vainqueur, à qui les deux Égypte sont soumises $» 40$.

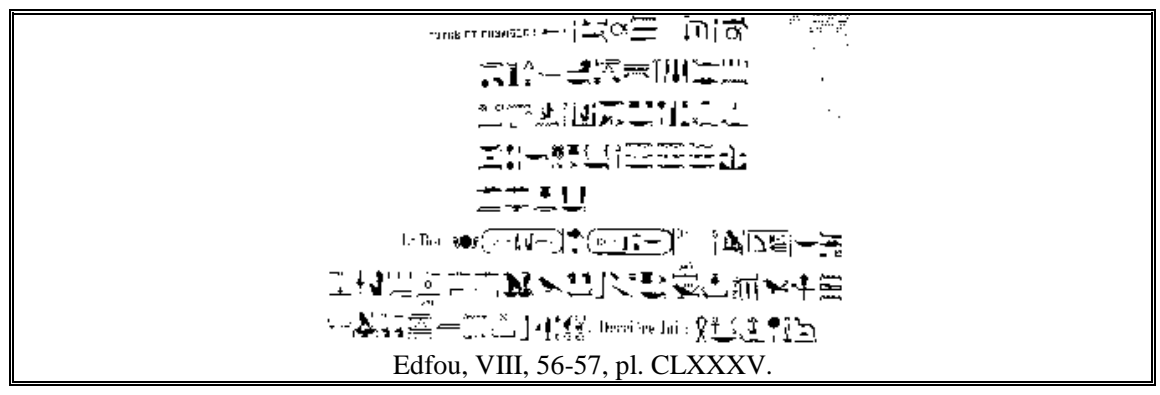

39 Edfou, VII, 275-276, pl. CLXXIV.

${ }^{40}$ Edfou, VIII, 56-57, pl. CLXXXV. 
Doc. $\mathbf{n}^{0} 10$.

Titre : di mAH n mAzxrw Dd mdw in : kAr.k @r dm Hnty xa Hm.k Hr st it.k pt Hr tp.k tA Xr rdwy.k pDwt-psDt dmD Xr Tbtyw.f m wsxt m tA m rk Hm nTrw Hay $n$ mAAk

«Présenter la couronne de la justification. Discours: Gloire à toi Horus aux cornes acérées, lorsque ta majesté apparaît sur le trône de ton père; le ciel est au-dessus de toi, la terre est sous tes pieds, et les Neuf Arcs sont réunis sous tes sandales dans la grande salle Wsxt, sur terre au temps de la majesté, les dieux se réjouissent de te voir ».

Le Roi : Wnn nswt bity Hr nst.f m(i) nsw n tAHr bn.f Hr ms wAH Hr siar mAzxrw Hr Hay ib n PsDt wab iqr Hm Hr rdi mAaxrw n aAAmAaxrw

«Or, le roi est sur son trône comme roi de la terre entière, il présente la couronne, il élève la justification, il réjouit le cour de l'ennéade. Il est un pur excellent, un prêtre de ... (?) en train de donner la justification au Grand Justifié» ${ }^{41}$.

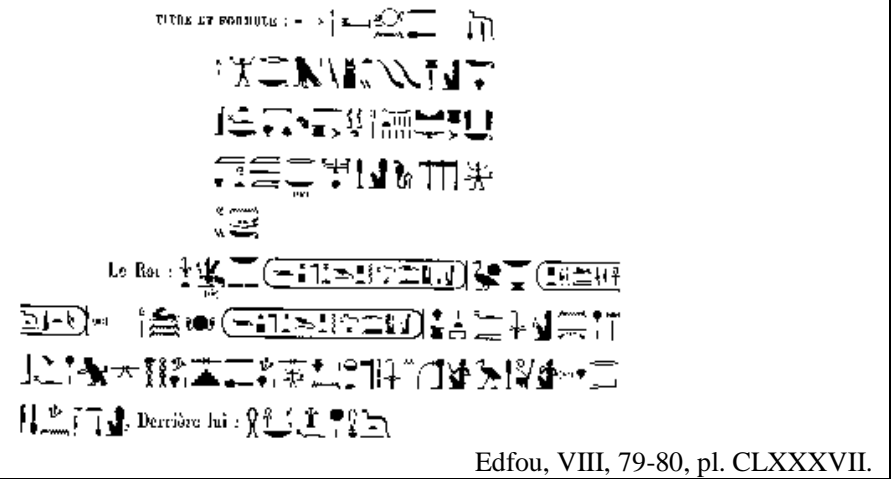

Doc. $\mathbf{n}^{0} 11$.

Titre : di mAH n mAaxrw Dd mdw mAH pn nfr iw HAt.k Ts n.k it.k it nTrw mAaxrw nxt. Tw Wsir DADAt dmD m wat

«Offrir la couronne de la justification. Discours : Cette belle couronne est sur ta tête, que ton père, le père des dieux a nouée pour toi. Vous êtes justifiés, vous qui suivez Osiris, Conseil réuni en un seul lieu ».

Le Roi : Wnn nsw Hr siwanst.f m ity nw Hsw Hr siar mH mAH ms mAarxrw Hr swDA Hr.n.swr sAnsw mi @r mama xftyw nit.f

«Or, le roi est en train de prendre l'héritage de son trône, en qualité de souverain d'Égypte, il présente la couronne, il offre la justification, il réjouit

${ }^{41}$ Edfou, VIII, 79-80, pl. CLXXXVII. 
(ainsi) la face du Grand Prince, car il est Horus, qui massacre les ennemis de son père» 42

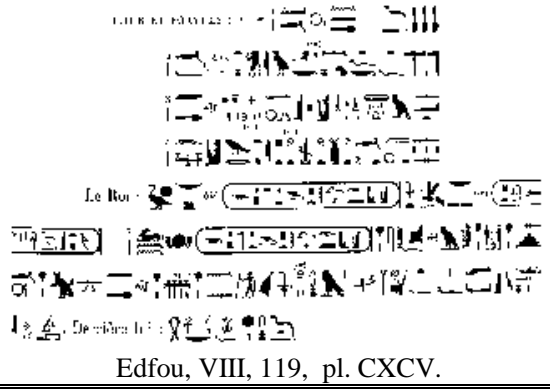

Doc. $\mathbf{n}^{0} 12$.

Titre : mAH r tp.k wTs nw tp.k it.k Tm sAk PsDt dmD Hr smAxrw pDwt dmD Xr rdwy.k it.k Wn nfr-ti mAaxrw Hr rdi n.k tAwy nTrw Hr ir m dp.k

«La couronne est sur ta tête pour qu'elle entoure ta tête. Ton père Atoum est ton protecteur, l'ennéade réunie te justifie, les Arcs sont réunis sous tes pieds. Ton père Onnophris le justifié te donne les deux Égypte, et les dieux montent la garde pour toi ».

Le Roi:anx nfr nTr Hm SmAat rdi tqr-pHty mi sA Ast wnt awy(.f)Xr mAH n mAaxrw rdi iAt.(s) n @r m xaf DAst.sn m smAStx nb xaw sARa

«Vive le bon dieu, le prêtre de Haute Égypte, dont la force est redoutable comme celle du fils d'Isis, qui étend les deux bras sous la couronne de la justification, qui donne (sa) fonction à Horus lorsqu'il apparaît, qui emmène les compagnons de Seth, Seigneur des couronnes, fils de $R^{c}, N \gg{ }^{43}$.

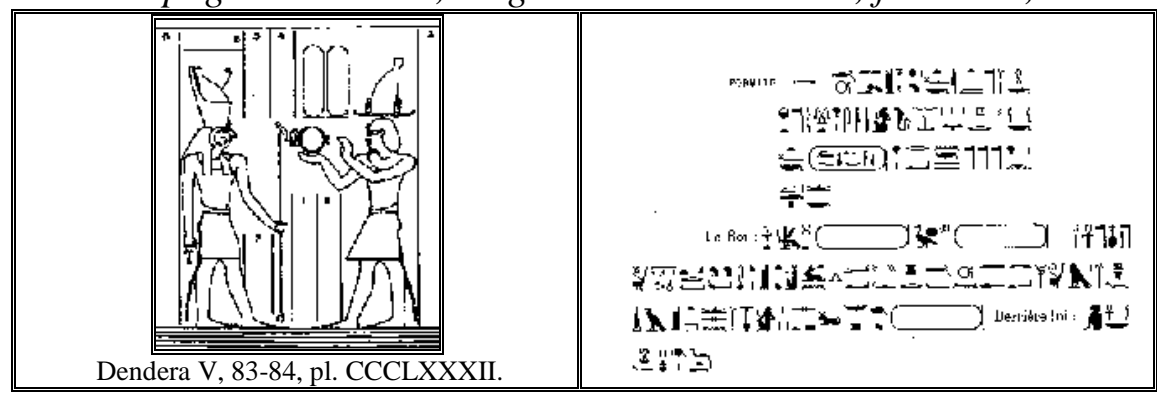

Doc. $\mathbf{n}^{0} 13$.

Titre : ms mAH n mAzxrw Dd mdw :Haj r.k/// [@r...] tpyw-apr Spst xaHm.k Hr st it.k hhn pt $m$ hhn tADr.f $m$ nTrw snwy $m$ nDm ib

${ }^{42}$ Edfou, 120, pl. CXCVI.VIII, 119-120, pl. CXCVI.

${ }^{43}$ Dendera V, 83-84, pl. CCCLXXXII. 
«(Apporter) la couronne de la justification. Discours: Gloire à toi [Horus...] qui préside au temple de la vénérable. Ta majesté apparait sur le trône de ton père, et le ciel acclame, la terre entière fait une ovation, les dieux ont le cour satisfait».

Le Roi : Nsw mnx mrw tA nb mAat int.f nDs wnn nsw Hr st.f m wnw nfr nb Haj mAH n iAbtt imntt Hr siar mDH n. nb.f mi nsw nD it.f iwan Wsir Ts tAwy nb m mAa$\mathrm{xrW}$

«Roi excellent qu'aiment ceux qui sont sur terre, Seigneur de la justice. Son abomination. C'est le péché. Or, le roi de Haute et de Basse Égypte est sur son trône comme un beau jeune homme, seigneur de joie, il présente la couronne à sa droite et à sa gauche, il élève la couronne vers celui à qui elle revient. Il est le sauveur de son père, l'héritier d'Osiris qui reçoit les deux Égypte entières en triomphateur» ${ }^{44}$.

\begin{tabular}{|c|c|}
\hline 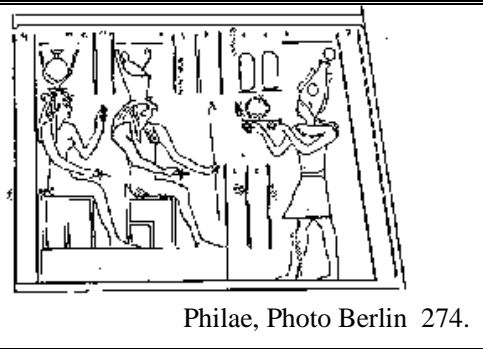 & 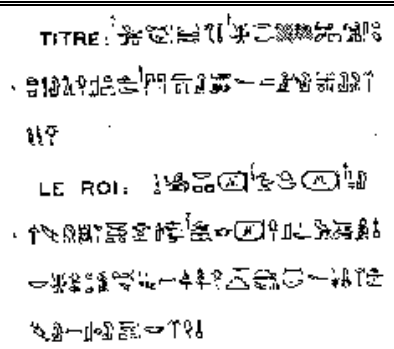 \\
\hline
\end{tabular}

Doc. $\mathbf{n}^{\mathbf{0}} 14$.

Titre : psD pt wDa sbAw.s xrs Snjt m pt pr.k m wpt mdw.k m mAa-xrw iT.k wrrt.k Hr nst G bb xr it.f Wsir Wnnfr mAaxrw

«Le ciel éclate, les dieux font chuter et chassent l'orage du ciel. Sors de ton procès ta voix est juste; la couronne de la justification est sur ta tête; tu reçois la couronne de Haute Égypte sur le trône de Geb, en existence de ton père, Osiris Onnophris le justifié ».

Le Roi : @r kA nxt HkA st Gbb Amm imyt -pr nt Wnnfr mAaxrw Snn @r sSp n MnTw nb WAst iwaw mnx $n$ ir $m$ W sir pXr n st $n$ Wnnfr mAa-xrw rnn n Ast $r$ HkA psSt nb xaw Ptwrmjs anX.w Dt mrj PtH

«Horus le taureau vigoureux, qui monarchie sur le trône de Geb, qui saisit les titres de propriété d'Onnophris le justifié le roi de Haute et de Basse Égypte, le portrait (figure) d'Horus, l'incarnation de Montou seigneur de Thèbes, bon hériter d'Osiris, hériter d'Onnophris le justifié, qu'Isis a élevé pour régner les deux parts, seigneur des couronnes, Ptolémée vivant éternellement, aimé de Ptah» ${ }^{45}$.

${ }^{44}$ Philae, Photo Berlin 274.

${ }^{45}$ CdE 30 , 268-269.Urk. VIII, inscr. $\mathrm{n}^{0}$ 20; St El H, p. 85-87.= 
1 - مراسات في اثار الوطن العربي

\begin{tabular}{|c|c|}
\hline 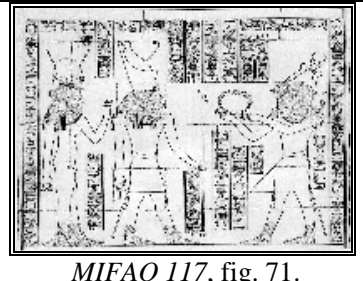 & 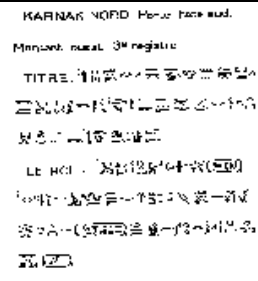 \\
\hline
\end{tabular}

\section{Osiris et la couronne de justification :-}

Les marques abydénienes de la couronne:-

- L'inscription de la statue (19 ${ }^{\mathrm{e}}$ dyn.), on lit : « Reçois la couronne (de justification) provenant de W-Pqr en tant que don du dieu grand (Osiris $^{46}$.

- Une inscription de la tombe de Sn-nfr s'exprime

di.n.f n.k wnHn mAaxrw «Reçois la couronne de justification» provenant de $\mathrm{W}-\mathrm{Pqr}^{47}$.

- Ainsi, dans le papyrus Louvre N 3129, on dit de Seth : «Tu es allé à rencontre des rebelles à W-Pqr, tu as pris (ou coupé ?) la couronne de justification ${ }^{48}$.

- Le Papyrus de la reine Nedjmet ${ }^{49}$ de la $21^{\mathrm{e}}$ dyn., qui contient un long rituel intitulé « apporter la couronne de la justification », dans la cérémonie de la fête Wag.

- Sur la statue du grand majordome de Memphis, Imenhotep ${ }^{50}$, de recevoir des bandelettes vertes ou rouges à l'occasion de la fête Wag.

$=$ Porte de Montou, face sud, extérieur, montant ouest, $3^{\mathrm{e}}$ registre.

S.H.Aufrère, Le propylône d'Amon-Rê-Montou à Karnak-Nord, MIFAO 117, 2000, 402-406, fig. 71. Ptolémée III Évergète apportant la couronne de la justification à Harendotès et à Isis.

${ }^{46}$ L.Keimer, Die Gartenpflanzen im Ägypten, I, Berlin 1924, 145.

J.Assmann, Das Grab des Basa (Nr. 389) in der thebanischen Nekropole, $A V$ 6,1973,110,fig. 38. P.P.Koemoth, op.cit., $246 \mathrm{n}^{\circ} 893$.

${ }^{47}$ L.Manniche, Lost Tombs. A Study of Certain Eighteenth Dynasty Monuments in the Theban Necropolis, Londres et New York, 1988, 118.

${ }^{48}$ Urk., VI, 133, 19-22. \& Ph.Derchain,op.cit., 237.

${ }^{49}$ W.Budge, Book of the Dead, Facsimiles of papyri of Hunefer, Anhai.,pl. 7-9. \&CdE 30, 235.

${ }^{50}$ J.Yoyotte, Les pèlerinages dans l'Égypte ancienne, dans : Sources Or. 3, (1960), 36. 
Le couronnement des morts, on le connait concernant au moins dès le Nouvel Empire, comme il ressort de la stèle du Louvre A $66^{51}$. in n.i mAH n mAaxrw

"Je t'apporte la couronne de la justification".

- Sur la stèle de l'architecte Hori ${ }^{52}$ de recevoir des bandelettes en cette même fête et du vœu d'Ankhefnenkhonsou ${ }^{53}$ de recevoir des étoffes de la main des deux sœurs à W-Pqr et une guirlande le jour de la fête Wag.

- D'après le chap. 169 du Livre des Morts, le matin de la fête Wag est la fête Sed d'Osiris, dont le triomphe et l'entrée dans l'assemblée divine de la Douat sont proclamés à ce moment. La couronne de justification lui est donnée et c'est sans doute à cette occasion que les acclamations ont lieu et que l'on voit le dieu ${ }^{54}$.

L'Ombite attrape la couronne destinée sans doute à Horus, s'affirmant ainsi comme le successeur conformité d'Osiris. Cela signifie-t-il que la couronne était fabriquée à partir de rameaux gardés sur un des arbres poussant près du tombeau osirien ${ }^{55}$.

Le symbolisme végétal surmontait à du reste très bien à ce cérémonial des grandes fêtes abydéniennes, qui mettent en scène le mythe de la résurrection du dieu ${ }^{56}$.

La fête Wag, bien attestée dans la littérature funéraire jusqu'à l'Époque Romaine ${ }^{57}$ est absente des inscriptions des temples

${ }^{51}$ CdE 30, 235.

${ }^{52}$ Urk. IV, 1947, 15.

${ }^{53}$ M.Aylward \& M.A.Blackman, « The funerary Papyrus of æenkefenkhons", dans: JEA 4, (1917), 124, pl. 26, col. 11 et 4.

${ }^{54}$ Budge, BD I, 104, début du chap. 19 (Papyrus de la reine Nedjemet.

À partir de la XXI ${ }^{\text {ème }}$ dynastie, le don de la couronne de justification à la fête Wag est bien attesté. $C d E$ 30, 238.

${ }^{55} \mathrm{Ce}$ sont les étiquettes démotiques du Musée du Louvre qui nous apportent une première preuve de l'utilisation de branches d'arbre, ici d'olivier, dans la confection de «bouquets de justification $(a n \times n D j) »$. L'existence de relation entre les bouquets et la justification est assurée. Ainsi l'étiquette Louvre AF 11169 précise : «... qu'on te (le défunt) donne un bouquet d'olivier de justification dans l'Occident (la nuit du) 25 au 26 Khoiak, alors que (tu) marches vers Osiris... ». P.Koemoth, op.cit., 35.

${ }^{56}$ CdE 30, 230-231.

${ }^{57}$ Papyrus Leiden I, 32; BM 10051, $15,4$. 
ptolémaïques et romains de sorte que l'on est en droit de se demander si le rite de la présentation de la couronne de justification, dans ses éléments abydéniens, n'est pas une autre façon de désigner la fête $\mathrm{Wag}^{58}$.

\section{L'offrande de la couronne de justification à Osiris dans les temples ptolémaïques et romains ${ }^{59}$ :-}

Doc. $\mathbf{n}^{0} 1$

Titre : Ts mAH n mAaxrw $r$ tp nw HAt.f $m D H$ hnn tp.f $m$ imjw.f

«Nouer la couronne de la justification autour de la tête de celui à qui elle appartient. Parer sa tête au moyen de ce qui est dedans».

Le Roi : ... it.f xftjw.f m nknw. f mAH pn nfr Dd mdw mn n.k mAH pfy nfr n mAa xrw Ts n.k it.k Tm mDH n sw m HAt.k tfy anx mryt nTrw anx Dt mAaxrw.k xr wrw DADAt.k

«...son (père). Ses ennemis sont en sa puissance. Discours : Je te présente cette belle couronne de la justification qu'a tressée pour toi ton père Atoum ; je l'ai posée sur ta tête pour que tu vives car les dieux aiment que tu vives à jamais. Tu es justifié devant le Grand Conseil » ${ }^{60}$.

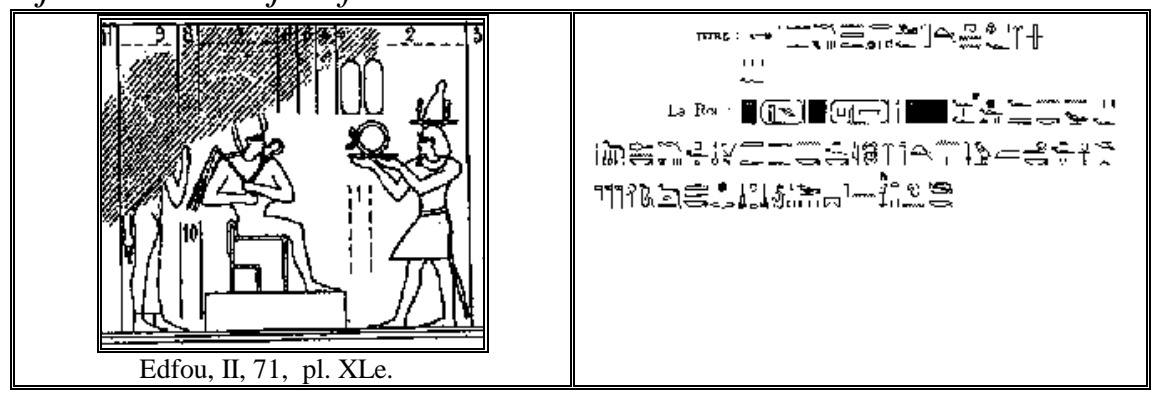

Doc. $\mathbf{n}^{\mathbf{0}} \mathbf{2}$

Titre : di mAH n mAaxrw. Dd mdw mn. k mAH pfy nfr $n$ mAaxrw wTs n.k it Tm nTrw dmD Hr rdit.n iAt.k P sDt Hr mAzxrw.k DADAt $n$ tmArdi n.k xaw.k iwan $\mathrm{G} b b \mathrm{dr}$ mryt $\mathrm{n} \mathrm{Hm}$

«Offrir la couronne de la justification. Discours. Accepte, je te prie, cette belle couronne de la justification, qu'a tressée pour toi (ton) père Atoum. Les dieux sont réunis pour te donner ta fonction, l'ennéade te justifie, le conseil de la natte te donne tes couronnes, tandis que l'héritage de Geb est gardé pur pour la majesté ».

${ }^{58}$ CdE 30, 234-238. \& P.P-Kriéger, «Wag Fest », dans : LÄ VI, 1136.

${ }^{59}$ CdE 30, 241-242.

${ }^{60}$ Edfou, II, 71, pl. XLe. 
Le Roi : nTr mnx Xrj-Hb n imj rnpt.s sAiwa n Gbb DADAt PsDt ///// Dd mdw iw.n.i $\mathrm{Hr}$.k tpj n G bb smAax xrw.f m DADAt siar n.k mDH Hr imntt iAbt di.k $r$ tp twt mAa xrw wnnfr mAaxrw tpj $n \mathrm{Gbb}$

« Le dieu excellent, le ritualiste de celle qui est en son année, fils héritier de Geb, pour qui le conseil de l'ennéade IIII. Discours : Je suis venu vers toi, ainé de Geb, qu'il a justifié en présence du conseil. Je t'ai présenté la couronne à gauche et à droite, et tu l'as posée sur la tête, de sorte que tu es justifié comme Onnophris le Justifié, l'ainé de Geb » ${ }^{61}$.

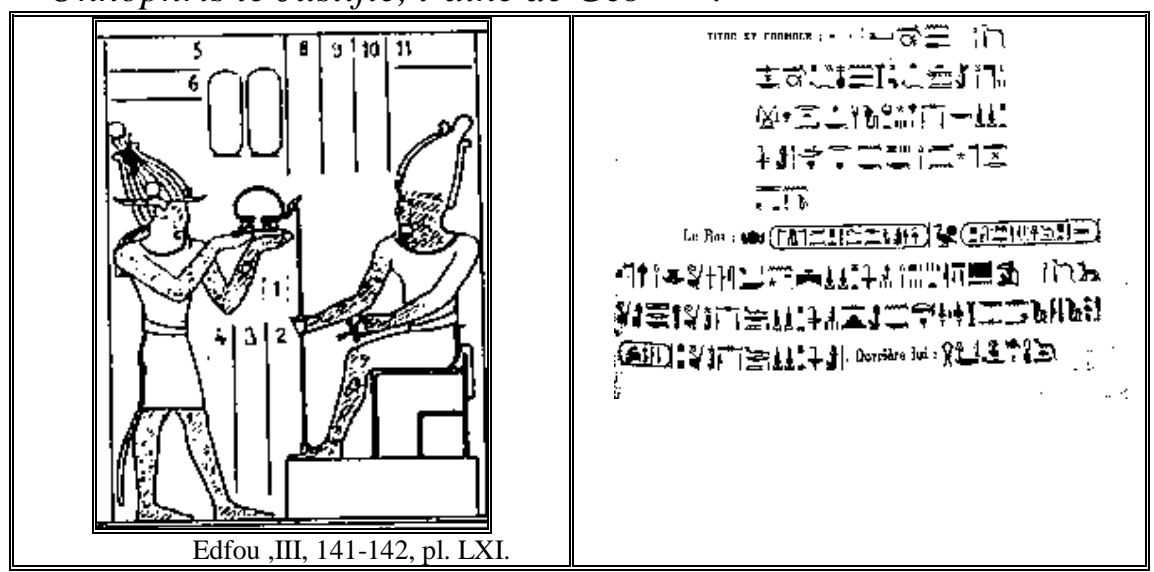

Doc. $\mathbf{n}^{\circ} 3$

Titre : Ts mAH n mAaxrw Dd mdw in mAH r tp.k mDH.n.f hnw tp.k swr.f Sfy $r$ nTrw it.k Tm Hr smAaxrw.k Gbb Hr rdit n.k iwa.f sA.k @r Hr sxri xftjw.k Hr wnDw ptpt n.k rkyw.k

«Nouer la couronne de la justification. Discours : La couronne est sur ta tête, elle pare ton crâne, elle rend ta dignité plus grande que celle des dieux. Ton père Atoum te justifie, Geb te donne son héritage, ton fils Horus renverse tes ennemis, il poignarde tes rebelles $» 62$.

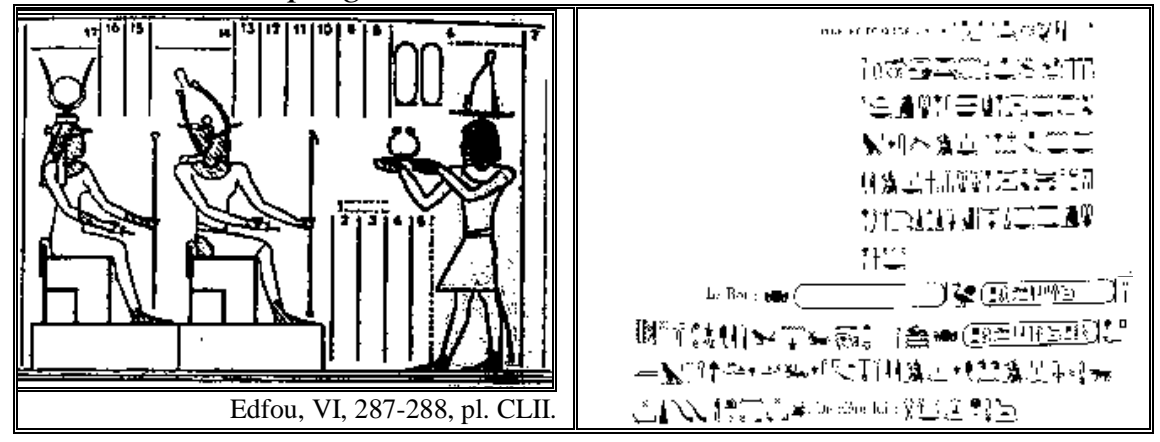

${ }^{61}$ Edfou, III, 141-142, pl. LXI.

${ }^{62}$ Edfou, VI, 287-288, pl. CLII. 
Doc. $n^{0} 4$

Titre : ms mAH n mAzxrw Dd mdw mn n.k mAH mDH n.f tp.k wTs nw tp.k tfj.f anxt it.k Tm Ts. n.f n tp.k Hknw.k m mAaxrw Haj r.k sAk Hr nst.k mAaxrw.k nn wn xtjw.k

«Apporter la couronne de la justification. Discours : Accepte, je te prie, la couronne pour qu'elle couronne ta tête, pour qu'elle orne ton front. Puisses-tu vivre! Ton père Atoum, il l'a nouée autour de ta tête afin que ta gloire en provienne, en triomphe. Gloire à toi, ton fils est sur ton trône tandis que tu es justifié et que tes ennemis n'existent plus ».

Le Roi : mwt nTr iw n Srj.k HkA....k wAH nfr n nb imyw mr nTrw mAAsn twt iwa Gbb, nsw tAwy Hrj-tp tAw

«Le dieu chéri de sa mère. Je suis venu à toi, souverain... La belle couronne est pour le seigneur de douceur que les dieux aiment de voir, ... l'héritier de Geb, le roi de l'Égypte, qui règne sur les pays " 63.

\begin{tabular}{|c|c|}
\hline 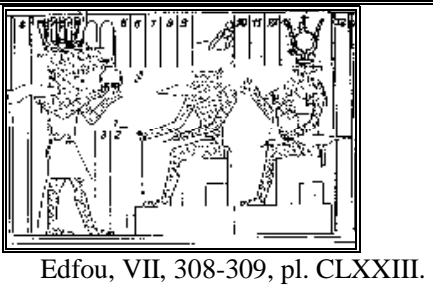 & 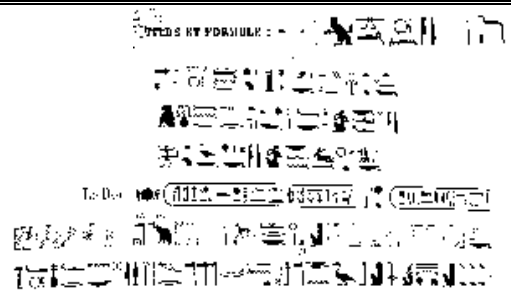 \\
\hline
\end{tabular}

Doc. $n^{0} 5$

Titre : di mAH n mAzxrw Dd mdw in mn.n tw mAH nfr n mAzxrw Ts n.Tn Tm it.f nTrw psDt dmD Hr iri bsATn sxmwt Hr nD Dt.Tn wDt n tw Gbb iwamntjwt Hr nst Tm DADAt dmD Hr smAaxrw. Tn Hr sHbn xrw $n$ xftyw. Tn wHm. Tn Hkn.wi $r$ fdw XAkwib.Tn $m$ sbt

«Offrir la couronne de la justification. Discours : Acceptez, je vous prie, la belle couronne de la justification qu'a nouée pour vous Atoum, le père des dieux, tandis que l'ennéade était réunie pour vous protéger, que les puissants protégeaient votre corps. Geb vous a transmis son héritage tandis qu'il est fermement établi sur le trône d'Atoum. Le conseil est réuni pour vous donner la justification et faire taire la voix de vos ennemis, pendant que vous répétez. votre ovation en son honneur par quatre fois, et que ceux qui sont révoltés contre vous sont à l'échafaud » ${ }^{64}$.

${ }^{63}$ Edfou VII, 308-309, pl. CLXXIII.

${ }^{64}$ Edfou, VI, 275-276, pl. CL. 

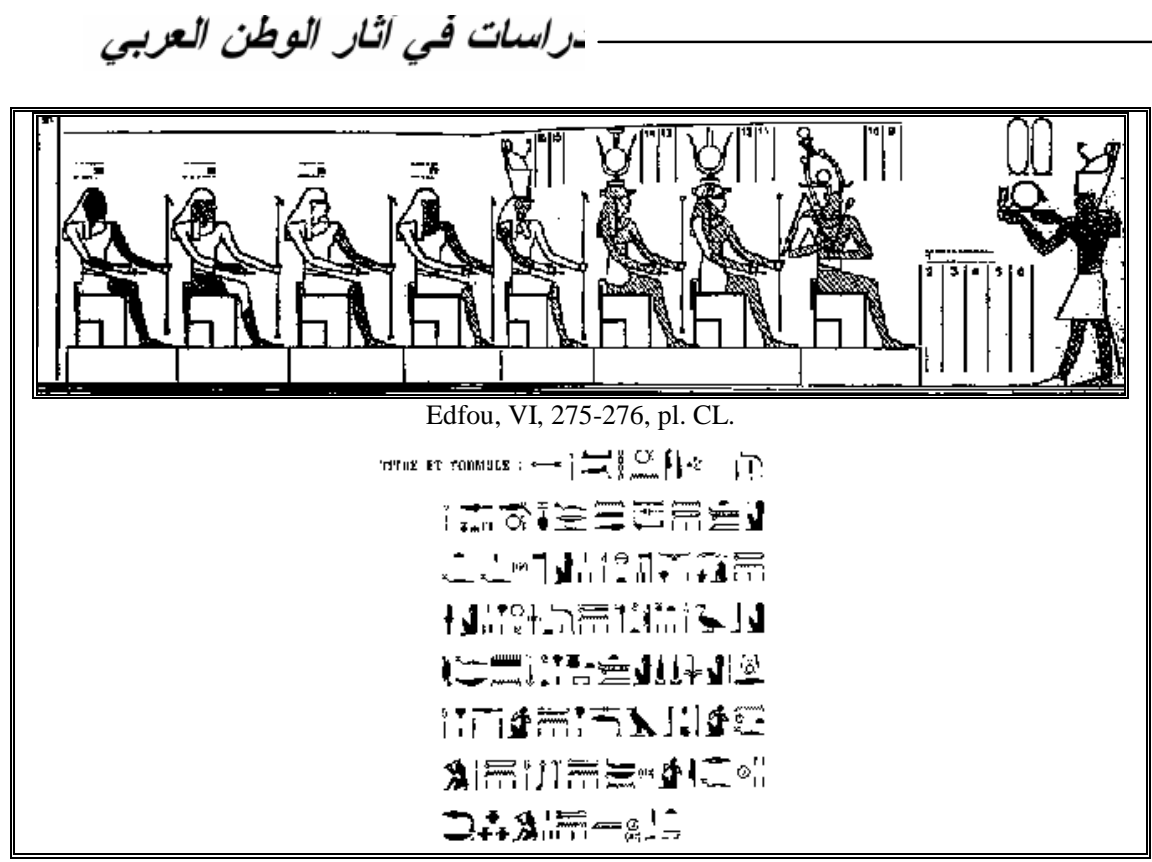

Doc. $n^{0} 6$

Titre : ms mAH n mAaxrw Dd mdw mAH pn nfr tp.k Ts n.k it.k it nTrw sHD.k tApn m twt.k anx.Tmrwt nTrw nbw mAAsn ...n Ra@r Axty sms.f Hr.k mDH Hknw iw r.k m mAaxrw

«Apporter la couronne de la justification. Discours : cette belle couronne est sur la tête qu'a nouée pour toi ton père des dieux, de façon que tu éclaires ce pays par ta tête. Puisses-tu vivre, car tous les dieux aiment de voir le... de $R^{c}$-Harakhty, lorsqu'il t'apporte la couronne. Acclamations à toi, en ta qualité de triomphateur ${ }^{65}$.

Edfou, IV, 259-260, pl. XC.

${ }^{65}$ Edfou, IV, 259-260, pl. XC. 
Doc. $\mathbf{n}^{0} 7$

Le Roi : ms mAH n mAz xrw : mAH pn nfr $r$ tp.k Ts n.k it.k it nTrw mAH pfy nfr n mAa -xrw apr.n.f hn.k anx.tw Dt mAaxrw.k n wn xftyw.k

«Présenter la couronne de la justification, cette belle couronne est sur ta tête, que ton père, le père des dieux a nouée pour toi. Cette belle couronne de la justification, elle orne ta tête pour que tu vives à jamais. Tu es justifié, est tes ennemis n'existent plus» ${ }^{66}$.

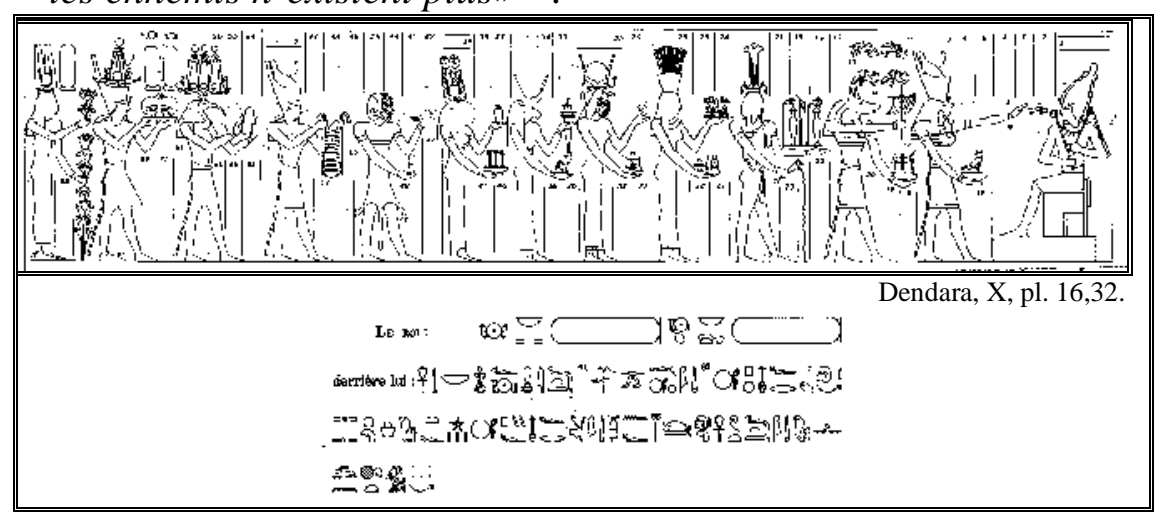

Doc. $\mathbf{n}^{0} 8$

Titre : Ts n.k it nTrw m mAH pfy nfr n mAaxrw m DADAt.k twy anxt mr(t) nTrw nbw [......]mAaxrw xrw.k xftyw. [k]bHn

"Nouer pour toi le père des dieux (=Ptah) t'attache cette belle couronne de justification sur cette tienne tête vivante qu'aiment tous les dieux [... ...], ta voix est victorieuse, [tes] ennemis sont abattus» ${ }^{67}$.

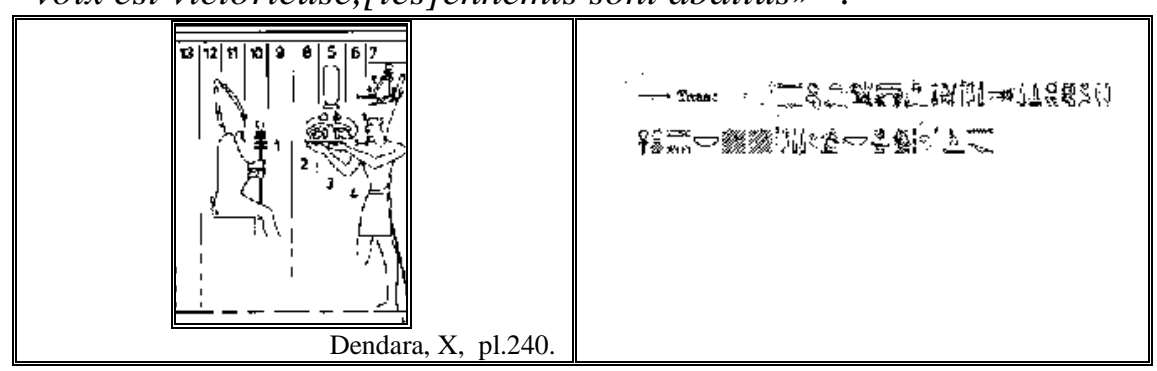

${ }^{66}$ Chapelles Osiriennes. LD IV, 57. PM 5, 94. Dendara, X, 35. pl. 16,32.

${ }^{67}$ Ibid., pl.240. 
Doc. $\mathbf{n}^{0} 9$

Titre : Ts mAH n mAzxrw Dd mdw mDH r tp.k mDH nw hn.k dr wr Styt nTrw «Nouer la couronne de la justification. Discours : la couronne est sur ta tête, elle orne ton crâne, pour que ta gloire soit plus grande celle des autres dieux ${ }^{68}$.

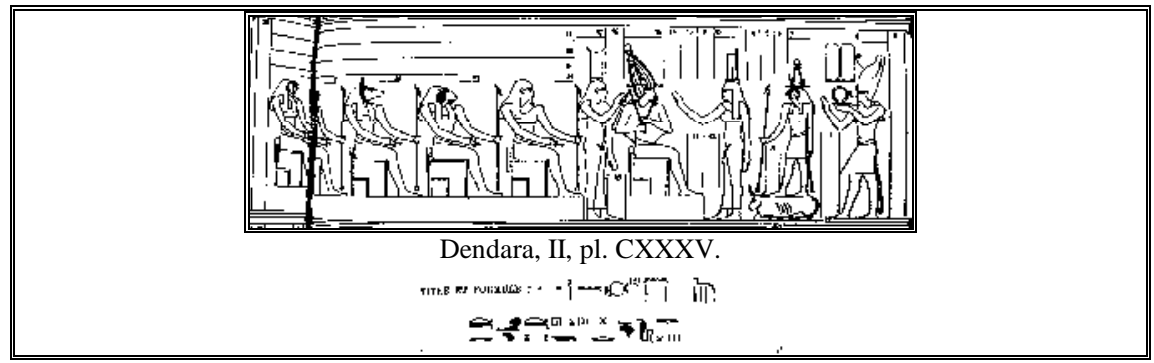

Doc. $\mathbf{n}^{\mathbf{0}} \mathbf{1 0}$

Titre : [Hnk mAH n mAz xrw]

Le Roi : nswt-bjtj jwan nTr.wj prj.wj stp.n ptH jrj mAtt-rasxm anx jmn sAra ptwlmys anx Dt mrj ptH $p(A) n T r m n x$

«Le roi de Haute et de Basse Egypte, héritier des dieux Epiphanes que Ptah a choisi qui fait la Maât de Rê image vivante d'Amon, le fils de Rêe. Ptolémée puisse-t-il vivre à jamais aimé de Ptah, le dieu Evergète ».

Ts.j n.k mAH pfj nfr n mAzxrw mj Ts n.k jt.k jtm jt nTrw

nTrw nb.(w) anx.k Dt jw smAa.n raHrw-Ax.tj xrw.k r (47) m HAt.k twj anx mrj

xftjw.k jw wD n.k jt.k Gbb jwa.f nb mj Hkn.k m mAaxrw mAaxrw.k r xftjw.k

«Je noue pour toi cette belle couronne de justification comme ton père Atoum, le père des dieux, la noue pour toi sur ce tien front de manière à ce que tu vives car tous les dieux aiment que tu vives à jamais. Rê-Harakhty a justifié ta voix contre tes ennemis. Ton père Geb ordonne pour toi tout son héritage. Viens pour être adoré en tant que justifié, parce que tu es justifié contre tes ennemis ${ }^{69}$.

${ }^{68}$ Dendara, II, 157, pl. CCXXVI.

${ }^{69}$ Temple d'Opet à Karnak, salle nord

C. Rochemonteix, Bibl.Égypt. III, 271-272 et pl. 12.

De Wit, Les inscriptions du temple d'Opet, à Karnak, Bibliotheca Aegyptiaca XI, 1958. 
1.

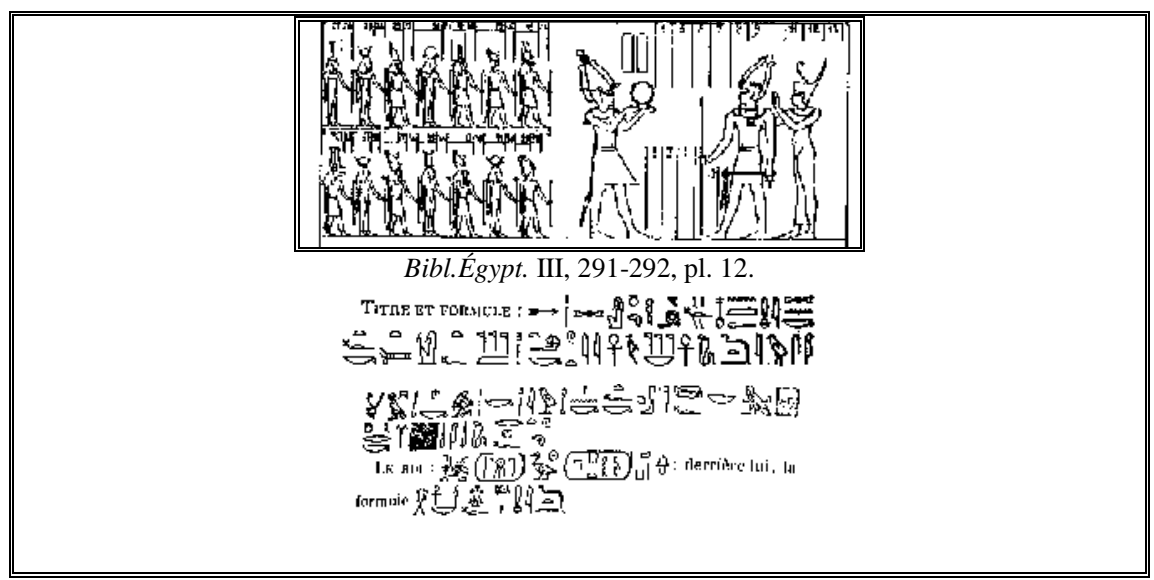

La couronne de la justification se présente à Harkhentekhtaï :-

L'offrande de la couronne de la justification se présente sous une forme spéciale lorsqu'elle est destinée à Harkhentekhtai ${ }^{70}$. Doc. $\mathrm{n}^{0} 1$

Titre: Ts mAH Dd mdw mAH pn nfr wi tp.k nb ... tpyw-akm-wr sA @r m sAk StAt Hr xw Dt.k Hry-tp ...( ) Hr Hbs n.k bHt

«Nouer la couronne de la justification. Discours : Cette belle couronne est autour de ta tête, Seigneur des (?), qui préside à Athribis. La protection d'Horus est ta protection; la déesse Chetat protège ton corps, Celle-qui-estsur-la tête (l'uraeus) monte la garde pour toi» ${ }^{71}$.

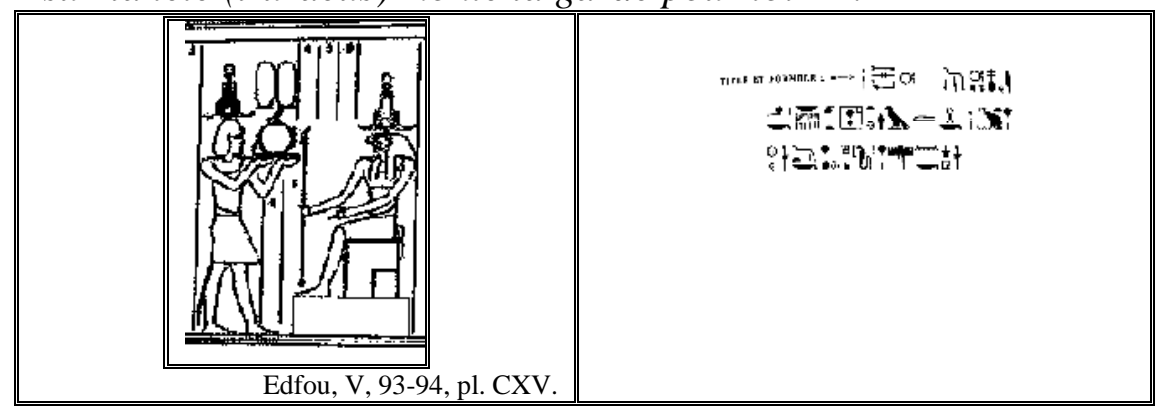

\footnotetext{
${ }^{70} \mathrm{Ce}$ dieu reçoit aussi une couronne appelé la couronne d'Horus. CdE 30, 243.

${ }^{71}$ Edfou, V, 93-94, pl. CXV.
} 
1 - راسات في اثثار الوطن العربي

Doc. $\mathbf{n}^{0} 2$

Le Roi : @r aA mAazrw nb nbty HA tp it.f Dd mdw mn.k mAH nfr mAaxrw Ts n.k sw $r$ Atf

«Horus le grand Justifié, le seigneur des deux déesses qui monte la garde derrière son père. Discours: Accepte, je te prie, cette belle couronne de la justification, je la noue pour toi autour de ton Atf ${ }^{72}$.

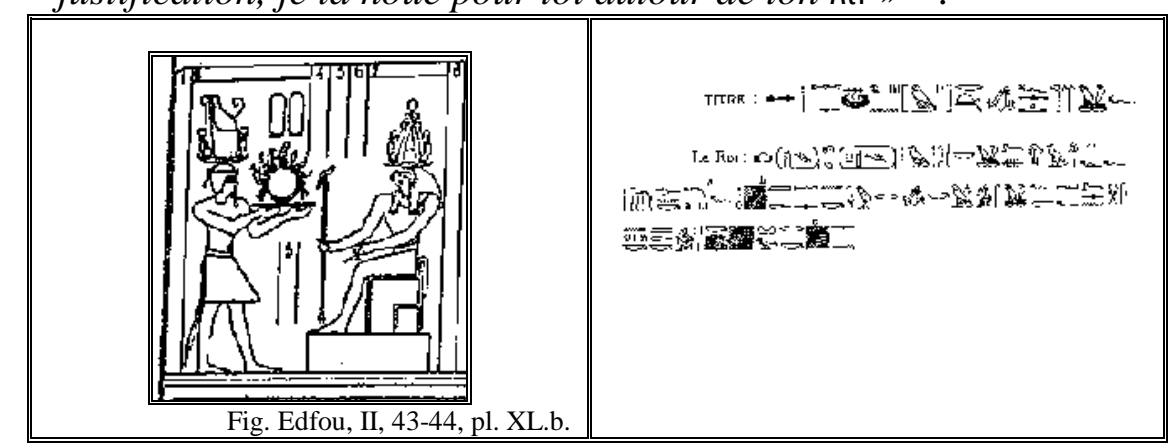

\section{La couronne de la justification offerte à Khépri :-}

Cette couronne est végétale er la met en rapport avec le soleil, pour souligner son caractère triomphant.

\section{Doc. $\mathbf{n}^{\mathbf{0}} 1$}

Titre : Di mAH n mAzxrw [n it.f Sps] mH tp.s m pr-a mAH pfy nn nfr m mAzxrw Ts $n$ r mDH tp.k mAaxrw.k m pt m tAxr DADAt wDa

«Consacrer la couronne de la justification à son père vénérable, couronner sa tête avec ce qui est dedans. Cette belle couronne de la justification, je la prends comme couronne chaque jour; tu es justifié au ciel, et sur la terre, auprès du Conseil qui juge» ${ }^{73}$.

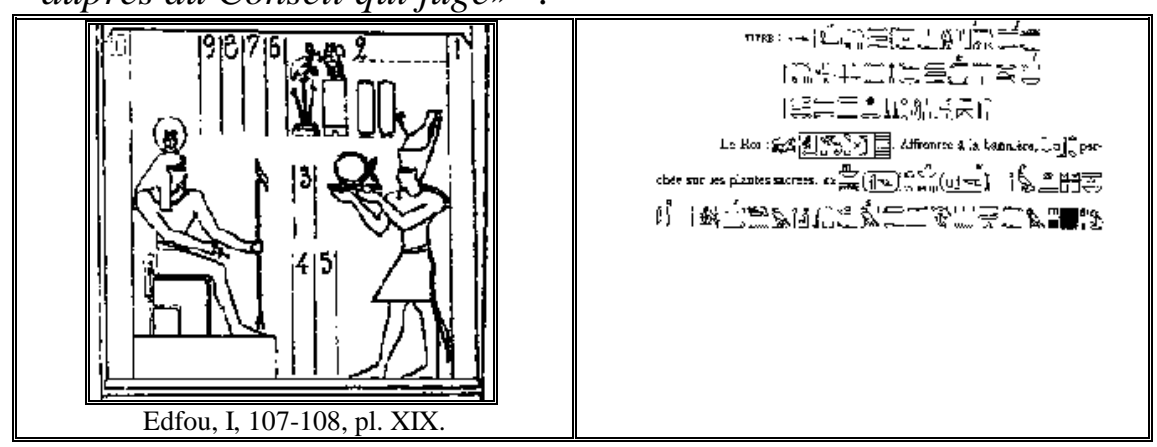

\footnotetext{
${ }^{72}$ Edfou, II, 43-44, pl. XL.b.

${ }^{73}$ Edfou, I, 107-108, pl. XIX.
} 
I · ماسات في اثثار الوطن العربي

\section{$\underline{\text { La couronne de la justification offerte à Thoth }}{ }^{74}$ :-}

Thot est «l'ordonnateur des paroles des dieux », ce qui rappelle le rôle qu'il a joué dans le procès d'Horus et de Seth, et son titre de « maître de la justice ».

\section{Doc. $\mathbf{n}^{0} 1$}

Titre : di mAH n mAaxrw Dd mdw mAH pn nfr $r$ tp.k Ts.n.k it.k it nTrw mAH n.k r.k m mAaxrw mAaxrw.k xftyw.k sxr

«Offrir la couronne de la justification. Discours : Cette belle couronne est autour de ta tête, que ton père Atoum le père des dieux a nouée pour toi. Gloire à toi en ta qualité de triomphateur, tu es justifié et tes ennemis sont renversés »

Le Roi : sA nsw n I sdn Dd mdw iw.n xr.k ib Ranw wDt mdw m nTrw in n.k mAH tfy nfr n mAamDH tp.k m nfrw twt nb mAat tpy-anTrw wr Sfyt xnt psDt

«Fils aîné d'Isden. Discours: Je suis venu auprès de toi, Couur de $R^{c}$, ordonnateur des sentences des dieux, je t'apporte Cette belle couronne de la justification pour qu'elle pare ta tête de sa beauté, comme le seigneur de la vérité, qui préside les dieux, dont la dignité est grande au sein de l'ennéade» ${ }^{75}$.

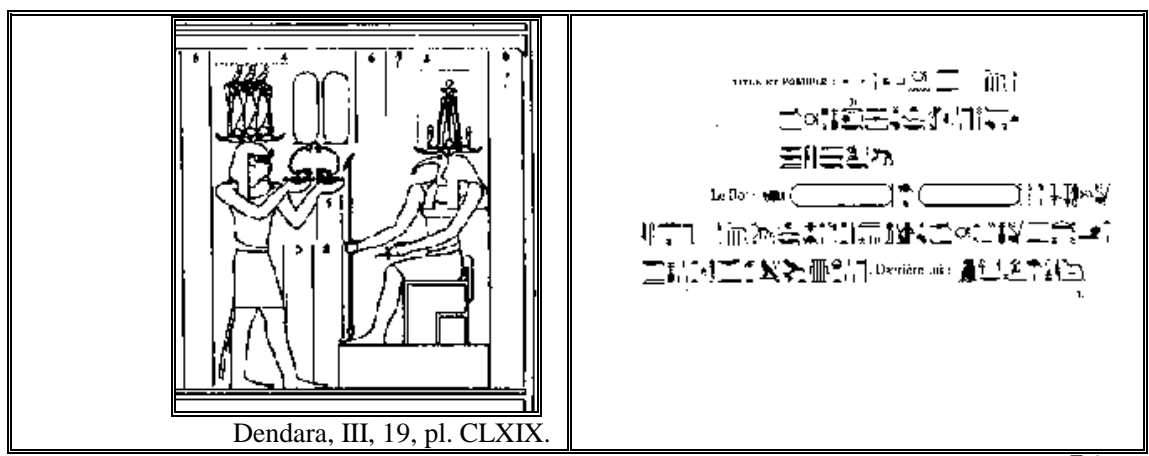

$\underline{L a}$ couronne de la justification offerte à Khnoum $^{76}$ :-

Doc. $\mathbf{n}^{0} 1$

Khnoum conduit de «Nebout» reçoit l'offrande rangé de la couronne et du bâton, avec les paroles habituels ${ }^{77}$.

\footnotetext{
${ }^{74}$ CdE 30, 249.

${ }^{75}$ Dendara, III, 19, pl. CLXIX.

${ }^{76}$ CdE 30, 249.

${ }^{77}$ Esne, colonne 14, Scène d'offrande sud.
} 

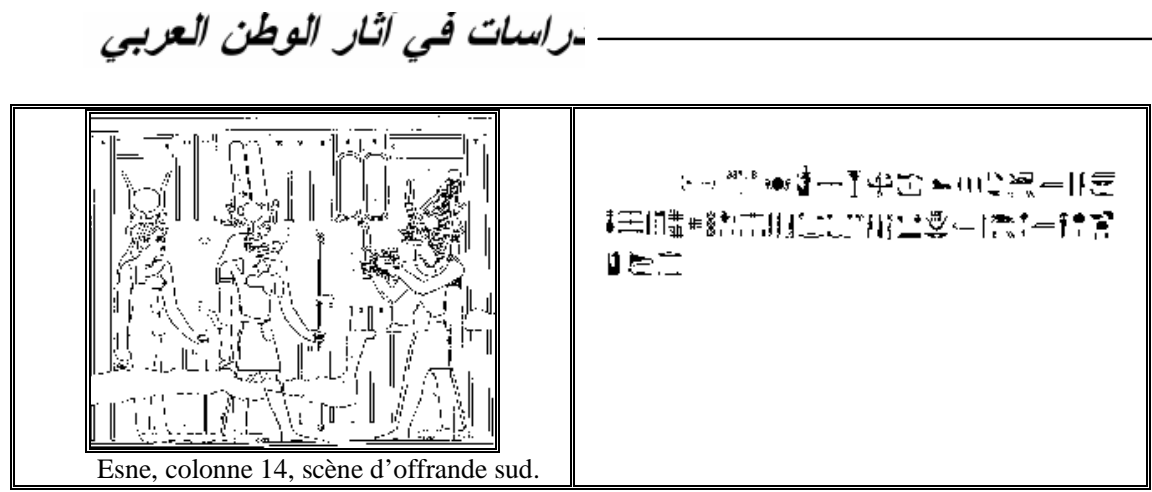

\section{La couronne de la justification offerte à Phior :-}

Le noyé Phior $(p A n . j r)^{78}$ assimilé à Osiris promet la vaillance au roi, assimilé à Horus fils d'Isis.

Doc. $\mathbf{n}^{\mathbf{0}} 1$

Titre: «Offrir la couronne à son père vénérable ».

Le Roi : «Roi de Haute et de Basse Égypte, Seigneur des deux pays, Pharaon. Fils de Ra, Pharaon».

Phior: «Discours de l'Osiris Phior: Je te donne la vaillance comme celle du Doc. $\mathbf{n}^{0} 2$ fils d'Isis ${ }^{79}$.

Titre: «Présenter la couronne à son père vénérable».

Le Roi : «Roi de Haute et de Basse Égypte,Fils de Ra,Pharaon» ${ }^{80}$.

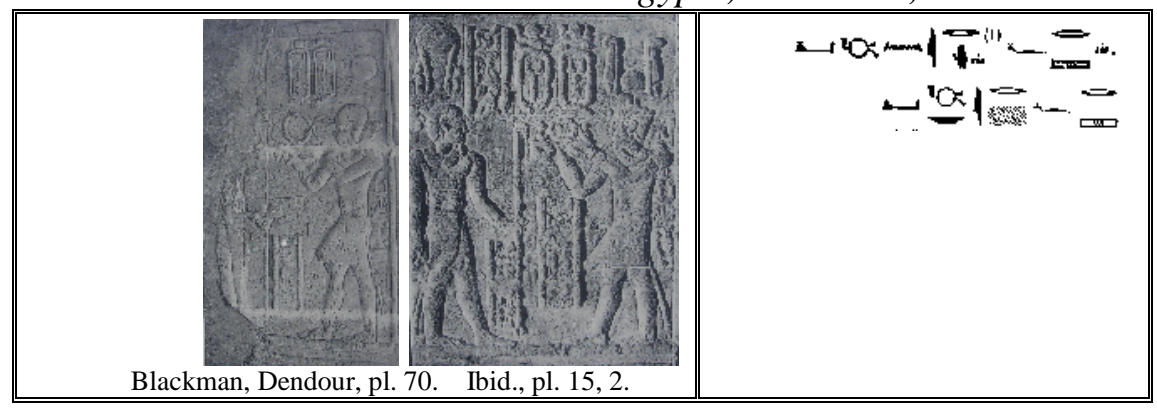

La couronne de la justification offerte au Pharaon de Biggeh ${ }^{81}$ :-

Le rite est de caractère nettement guerrier que le Pharaon de Senmet a été associé à sa célébration dans un des textes Philae. Ce

\footnotetext{
${ }^{78}$ (pAn.j r) LGG III, 17.

${ }^{79}$ Blackman, Dendour, p.45 et pl. 70, 2. Il s'agit ici de l'offrande d'une couronne ornée d'un seul uraeus à un mort divinisé, un noyé.

${ }^{80}$ Blackman, op.cit., 8, pl. 15,2.

${ }^{81}$ CdE 30, 250.
} 
personnage est bien connu par les tableaux de Philae et des temples de Nubie, où il reçoit diverses offrandes.

Doc. $\mathbf{n}^{0} 1$

Titre : « Gloire à toi, Horus( ?) de ...(à) la place de ton père ».

Le Roi : SAnsw m N xbt... wr pHty wdnw ... nfr tpj mrwt Ts mAH n mAzxrw n mAz xrw $n$ it.f aAmAzxrw...smAn hn r..f ... n Hm.f nTr-anHH

«Fils ainé de Nekhbet, premier né de ... (le héros ?) à la grande puissance, très redoutable, le beau jeune homme dont l'amour est doux, qui noue la couronne de la justification pour son père le grand justifié, qui l'unit à sa tête ... pour sa majesté, à jamais » ${ }^{82}$.

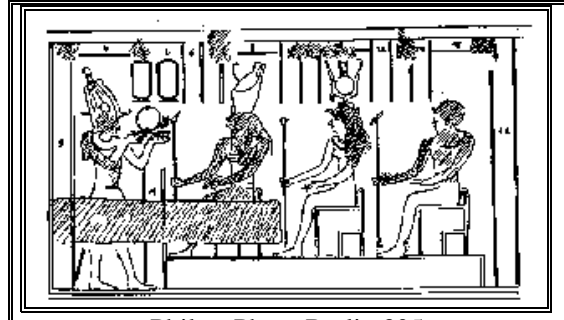

Philae, Photo Berlin 305

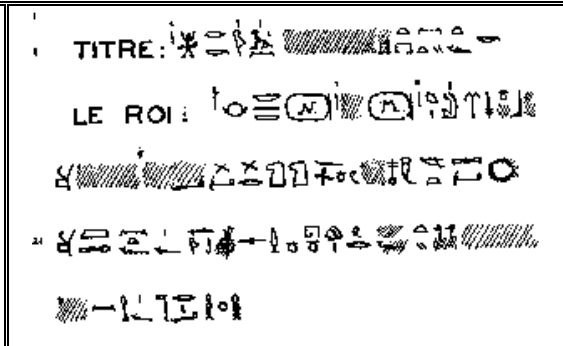

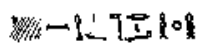

\section{La couronne de la justification offerte à Hathor ${ }^{83}$ :-}

Cette scène de Dendera porte un faux titre, car, dès la seconde phrase, on s'aperçoit qu'il s'agit de la couronne d'or, et non de celle de la justification.

Doc. $\mathbf{n}^{0} \mathbf{1}$

Titre : ms mAH n mAzxrw . Dd mdw ... mAH n nwb....

«Présenter la couronne de la justification. Discours ... la couronne d'or... en train de la nouer de ses deux mains... de la présenter ... ${ }^{84}$.

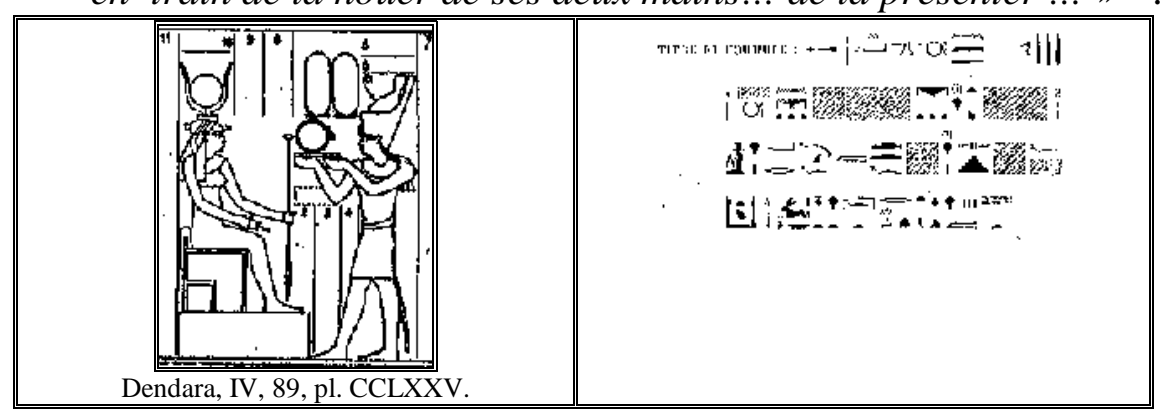

\footnotetext{
${ }^{82}$ Philae, Photo Berlin 305.

${ }^{83}$ CdE 30, 250.

${ }^{84}$ Dendara IV, 89, pl. CCLXXV.
} 
1 · مراسات في اثار الوطن العربي

\section{La couronne de la justification offerte à Chonsou :-}

Doc. $\mathbf{n}^{0} 1$

Titre : rdit n.k mAH pfy nfr $m$ mAaxr

« Je te donne cette belle couronne de la justification».

Le Roi :«Dieu onéreux de sa mère, Horus... taureau puissant,seigneur de...» ${ }^{85}$.

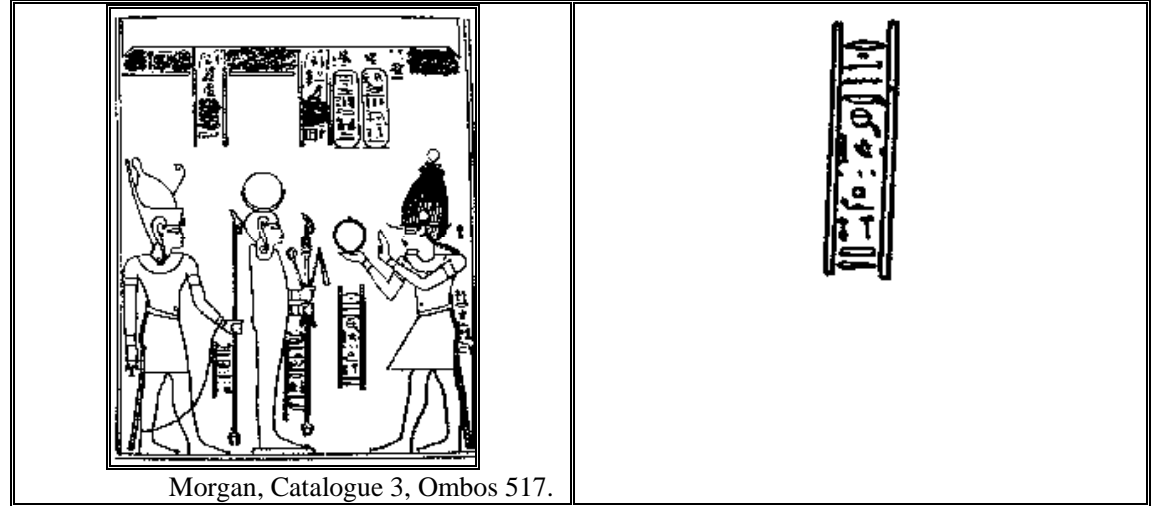

La couronne de la justification offerte à Mrwr ${ }^{86}$ :-

Doc. $\mathbf{n}^{0} 1$

Titre : Ts.n mAH n mAzxrw Hrj tp.k di n.k M rwr

«Noué la couronne de la justification autour de ta tête je te donne pour toi Mrwr $\gg 87$.

\begin{tabular}{|c|c|}
\hline 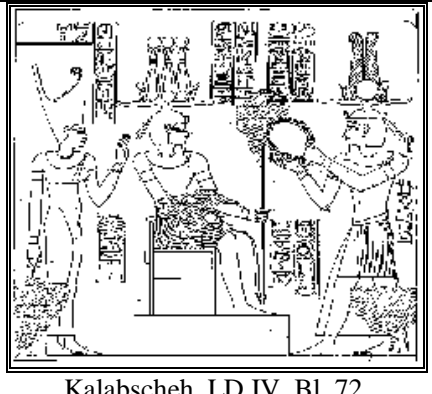 & 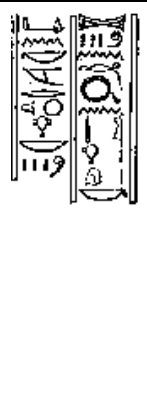 \\
\hline
\end{tabular}

\footnotetext{
${ }^{85}$ Morgan, Catalogue 3, Ombos 517.

${ }^{86}$ Mandulis, J.G.Griffiths, dans : L̈̈ III, 1177-1179. LGG III, 356.

${ }^{87}$ Kalabscheh, LD IV, B1. 72.
} 


\begin{tabular}{|c|c|c|}
\hline $\begin{array}{l}\text { On conclure d'a } \\
\text { couronne }^{88} \text { :- }\end{array}$ & ès les $\frac{\text { Conclusio }}{\text { exemplair }}$ & les divers formes \\
\hline Le type & La description & Les documents \\
\hline $\mathrm{n}^{\mathbf{0}} 1$ & $\begin{array}{l}\text { Le plus simple, un } \\
\text { bandeau noué. }\end{array}$ & $\begin{array}{c}\text { Chapitre } 19 \text { de livre de mort. } \\
\text { Edfou, pl. XXVI a. } \\
\text { Edfou, pl. XLe. } \\
\text { Edfou, pl. XIX. } \\
\text { Kom Ombo, } 517 . \\
\text { Philae, Photo } 305 . \\
\text { Esne, Colonne 14. } \\
\text { Edfou, pl. CLXXIV. } \\
\text { Kalabscheh, LD IV, Bl. } 72 .\end{array}$ \\
\hline$\overline{n^{0} 2}$ & $\begin{array}{l}\text { Le bandeau sans anneau } \\
\text { est orné de deux wDAt } \\
\text { opposées. }\end{array}$ & $\begin{array}{l}\text { Edfou, pl. XVI. } \\
\text { Edfou, pl. LXI. }\end{array}$ \\
\hline$\overline{n^{0} 3}$ & $\begin{array}{l}\text { Le type II, mais décoré } \\
\text { des plantes de Haute et } \\
\text { de Basse Égypte } \\
\text { opposées, soit à l'opposé } \\
\text { des wDAt, soit aux bouts. }\end{array}$ & $\begin{array}{c}\text { Edfou, pl. XXVI a. } \\
\text { Edfou, pl. CVI. } \\
\text { Edfou, pl. XC. } \\
\text { Dendara, X, pl. 16,32. } \\
\text { Dendara, X, pl.240. } \\
\text { Dendara,III, pl.CLXIX. }\end{array}$ \\
\hline$n^{0} 4$ & $\begin{array}{l}\text { Le bandeau simple, noué } \\
\text { ou non, avec deux uraeus } \\
\text { couronnés. }\end{array}$ & $\begin{array}{r}\text { Edfou, pl. CLXXXVII. } \\
\text { Dendera,V,pl.CCCLXXXII. } \\
\text { Edfou, pl. CXCVI. } \\
\text { Dendour, pl. 15, } 2 .\end{array}$ \\
\hline $\mathrm{n}^{0} 5$ & $\begin{array}{l}\text { Le type III, avec deux } \\
\text { uraeus couronnés. }\end{array}$ & $\begin{array}{l}\text { Philae, Photo Berlin } 274 . \\
\text { Philae, Photo Berlin } 305 .\end{array}$ \\
\hline $\mathrm{n}^{0} 6$ & $\begin{array}{c}\text { Un bandeau à sept } \\
\text { gonflements qui } \\
\text { paraissent être des } \\
\text { anneaux partagés de part } \\
\text { et d'autre des plantes de } \\
\text { Haute et de Basse Égypte } \\
\text { opposées et entourant un } \\
\text { œil wDAt. }\end{array}$ & Karnak Nord. \\
\hline $\mathrm{n}^{\mathbf{0}} \mathbf{7}$ & $\begin{array}{l}\text { Le bandeau simple, noué, } \\
\text { avec une seule uraeus } \\
\text { sans couronne. }\end{array}$ & Dendour, pl. 70, 2. \\
\hline
\end{tabular}

${ }^{88} C d E$ 30, 225-227. 
Les couronnes de la justification seraient supplémentaires en le sens qu'ils forment une image de l'éternité convenue après la victoire sur le mal. Ils permettent au mort d'être reconnu puis accueilli par Osiris dans son royauté.

Il s'agit d'un rite d'origine funéraire comme l'existence du chapitre 19 du Livre des Morts et les couronnes de momies.

Le caractère de mAzxrw s'applique à $\mathrm{R}^{\mathrm{c}}$ lui-même lorsqu'il triomphe de son ennemi Apophis.

Le rituel de la justification, qui domine pendant la présentation de la couronne, symbole du nouveau caractère de victoire l'épreuve du jugement, n'est qu'une scène qui assure le royaume à Horus et la réunification des deux Égypte, à la suite de la décision du grand conseil des dieux réuni à Héliopolis.

Quand Osiris est sorti de son procès et qu'il a été justifié, tandis que la couronne de la justification était sur la tête, Les dieux ont chassé et repoussé les nuages du ciel et que tu prenais la couronne d'Égypte sur le trône de Geb.

La présentation de la couronne de la justification symbolisait le triomphe du dieu contre ses ennemis, il semble que le caractère guerrier du rite lui ait donné une certaine valeur apotropaïque, qui s'exprime aussi dans les ornements posés sur la couronne, les discours eux-mêmes sont pleins de déclarations agressives, annonçant la victoire contre les ennemis sous à l'avantage du dieu, soit comme la parure du roi pour avoir accompli les rites.

Les scènes d'offrande est régulièrement semblable: le roi qui s'identifie généralement à Horus et est recommandé par une titulature spéciale offre la couronne au dieu suivit par les épithètes pour protéger le roi de sa protection, et pour attester une longue vie et pour la défense contre ses ennemis. Rarement une autre divinité participe à la cérémonie sans y participer autrement qu'en garantissant les paroles des personnages. Un troisième personnage régulier apparaît dans ces représentations d'offrande (le titre et la 
formule Ts nouer, ms apporter di , Hnk présenter, offrir) qui montre la nature de l'offrande et la formule de protection.

Le dieu, après avoir reçu la couronne du roi, en le priant de se la mettre sur la tête. Cette couronne de l'année précédente qui est ainsi remplacée de la tête du dieu à celle du roi en caractère de $s f x$ « placement en bas ».

\section{Sommaire}

La couronne et la guirlande de la justification «mAH n mAaxrw » plaçait sur le corps, le cou et la tête du défunt, cette ornementation florale reçut un caractère religieux ou symbolique.

La couronne symbolise le nouvel caractère de celui qui a remarqué victorieusement l'expression du jugement, dans le chapitre $19 \mathrm{du}$ Livre des Morts.

Le rite ptolémaïque d'offrande de la couronne de la justification se présente sous une forme complexe, où entrent des éléments qui appartient à Horus et à Osiris.

La couronne de la justification offerte à d'autres dieux par exemple à Harkhentekhtaï, à Khépri, à Thoth, à Khnoum, à Khnoum, au Pharaon de Biggeh, à Phior, à Hathor, à Chonsou, et à Mandulis.

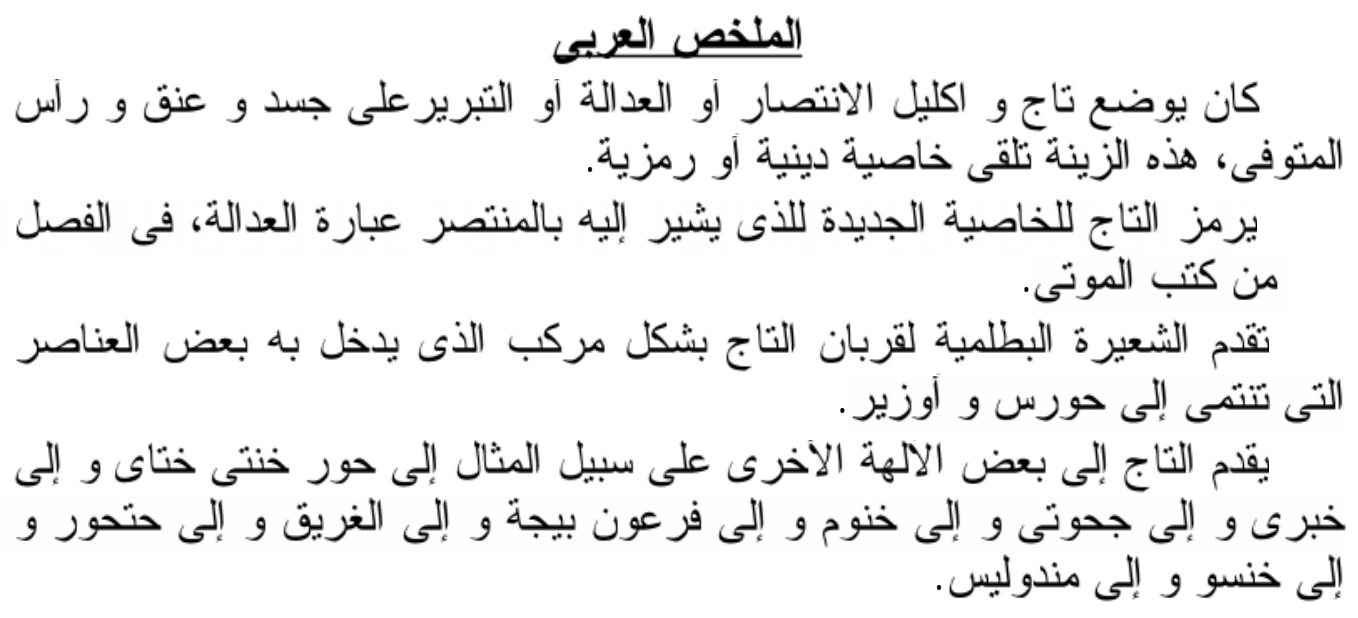

